\title{
Quantifying biologically and physically induced flow and tracer dynamics in permeable sediments
}

\author{
F. J. R. Meysman ${ }^{1}$, O. S. Galaktionov ${ }^{1}$, P. L. M. Cook $^{2, *}$, F. Janssen ${ }^{2}$, M. Huettel ${ }^{3}$, and J. J. Middelburg ${ }^{1}$ \\ ${ }^{1}$ Centre for Estuarine and Marine Ecology (CEME), The Netherlands Institute of Ecology (NIOO-KNAW), Korringaweg 7 , \\ 4401 NT Yerseke, The Netherlands \\ ${ }^{2}$ Max Planck Institute for Marine Microbiology, Celsiusstr. 1, Bremen, 28359, Germany \\ ${ }^{3}$ Department of Oceanography, Florida State University, Tallahassee, FL 32306-4320, USA \\ *now at: CSIRO Land and Water, 120 Meiers Rd Indooroopilly, 4075, Qld, Australia
}

Received: 20 November 2006 - Published in Biogeosciences Discuss.: 8 December 2006

Revised: 2 August 2007 - Accepted: 8 August 2007 - Published: 9 August 2007

\begin{abstract}
Insight in the biogeochemistry and ecology of sandy sediments crucially depends on a quantitative description of pore water flow and the associated transport of various solutes and particles. We show that widely different problems can be modelled by the same flow and tracer equations. The principal difference between model applications concerns the geometry of the sediment-water interface and the pressure conditions that are specified along this boundary. We illustrate this commonality with four different case studies. These include biologically and physically induced pore water flows, as well as simplified laboratory setups versus more complex field-like conditions: [1] lugworm bio-irrigation in laboratory set-up, [2] interaction of bioirrigation and groundwater seepage on a tidal flat, [3] pore water flow induced by rotational stirring in benthic chambers, and [4] pore water flow induced by unidirectional flow over a ripple sequence. The same two example simulations are performed in all four cases: (a) the time-dependent spreading of an inert tracer in the pore water, and (b) the computation of the steady-state distribution of oxygen in the sediment. Overall, our model comparison indicates that model development for sandy sediments is promising, but within an early stage. Clear challenges remain in terms of model development, model validation, and model implementation.
\end{abstract}

\section{Introduction}

Sandy sediments make up a substantial part of coastal and shelf areas worldwide, and consequently, they form an important interface between the aquatic environment and the earth surface (Huettel and Webster, 2001). A prominent characteristic of sandy sediments is their high permeability, which allows pressure gradients to force a flow through the

Correspondence to: F. J. R. Meysman

(f.meysman@nioo.knaw.nl) pore spaces. This pore water flow forms an effective transport mechanism for various abiotic components, like solutes (Webb and Theodor, 1968) and fine clay particles (Huettel et al., 1996), but also acts as a vector for biological particles, such as bacteria and algae (Rusch and Huettel, 2000). Previous studies have shown that advective transport exerts a major control on sediment biogeochemistry (e.g. Forster et al., 1996; Shum and Sundby, 1996), microbial ecology (e.g. de Beer et al., 2005), and solute exchange across the sediment-water interface (e.g. Huettel et al., 1998). Therefore, our understanding of the biogeochemistry and ecology of sandy sediments crucially depends on a quantitative description of pore water flow and the associated transport of various solutes and particles.

The pressure gradients that drive pore water flow in sandy sediments are generated by physical and biological factors. Physically driven pore water flow may result from wave action leading to oscillatory pressure fields and currents (Riedl et al., 1972; Precht and Huettel, 2003) or from unidirectional bottom currents interacting with the topography of the sediment-water interface (Thibodeaux and Boyle, 1987). This bottom topography may have a pure physical origin, such as sand ripples induced by currents, or can be created by biology, such as the pits and sediment mounds of various benthic organisms, like mud shrimp, lugworms and rays (Huettel and Gust, 1992b; Ziebis et al., 1996). Yet, pore water flow may also be directly induced by burrowing organisms, a process typically referred to as bio-irrigation (Aller, 2001; Meysman et al., 2006a). To sustain an aerobic metabolism, benthic fauna flush their burrow structures with oxygen-rich water from the overlying water column (Gust and Harrison, 1981; Webb and Eyre, 2004). In sandy sediments, water may be actively pumped across the burrow wall, thus generating pore water flows in the sediment surrounding the burrow (Foster-Smith, 1978; Meysman et al., 2005). Such advective bio-irrigation significantly alters the flux, consumption and distribution of various solutes within

Published by Copernicus Publications on behalf of the European Geosciences Union. 
the pore water (Timmermann et al., 2002; Meysman et al., 2006a, b).

In recent years, mathematical modeling has proven a valuable tool to quantify in-situ flow patterns in sandy sediments and assess their biogeochemical effects (e.g. Rena and Packman, 2004; Meysman et al., 2005). Partly, this is because the experimental characterization of solute transport in sandy sediments is challenging for a number of reasons. Firstly, the flow pattern is three-dimensional, while pore pressure and tracer concentrations are typically assessed by point measurements. Even with novel tracer imaging techniques, based on arrays of point measurements, it remains very difficult to constrain the flow pattern (Precht and Huettel, 2004; Reimers et al., 2004). A second problem is that tracer measurements cannot be performed without inflicting a significant disturbance to the sediment. The insertion of electrodes, planar optodes, benthic chambers or other measuring devices into sandy sediment implies that artificial obstacles are introduced for the pore water flow and/or that the overlying currents are disturbed. Such a change in boundary conditions might drastically alter the flow pattern within the sediment. Thirdly, the pressure fields that induce pore water flow in sandy sediments are very dynamic by nature. Key physical forcings such as sediment topography, waves and currents can vary dramatically over short time scales (Wheatcroft, 1994; Li and Amos, 1999; Packman and Brooks, 2001). A similar variability also characterizes biologically-induced flow, where bio-irrigating organisms may change their position and adapt their pumping rate over time scales of minutes to days (Kristensen, 2001). Obtaining representative measurements under all likely combinations of these forcings is virtually impossible. Faced with such experimental difficulties, modelling provides a complementary research tool. Models can be used to interpret tracer data obtained from experiments, to assess potential distortions introduced by measuring devices, or to examine complex field-like conditions that cannot be reproduced in the laboratory.

Nonetheless, models are far from being used as a routine tool in biogeochemical studies of sandy environments, and on the whole, model development is still within an early stage. One aspect that needs attention is the comparison and integration of the existing model approaches for physically and biologically induced solute transport. As noted above, in near-shore sands, both types of processes are important, and may even interact (see below). Typically however, biological and physical aspects have been studied in different research communities. Physical mechanisms have attracted the attention of engineers and physicists, focusing on the waveinduced advection below sand ripples (Shum, 1992) and flow over ripples (Savant et al., 1987; Rutherford et al., 1995; Elliott and Brooks, 1997a). As a result of this, a firm modelling tradition has been established in the engineering literature (e.g. Rena and Packman, 2004; Cardenas and Wilson, 2006). In contrast, modelling approaches to biologically induced flow have only been recently explored, in particular by marine biogeochemists focusing on the effects of irrigational flows induced by sediment-dwelling organisms (Meile et al., 2003; Meysman et al., 2005, 2006a, b). Overall, these model studies clearly demonstrate the potential of numerical modeling to further our understanding of advective transport in sandy sediments. However, model applications differ in the software that is used, the model approximations that are incorporated, the boundary conditions implemented, and even in the basic model equations (e.g. Darcy versus Brinkman formulation; see discussion below). These differences make cross-system comparisons difficult.

Therefore, it would be valuable to apply a single model approach to different mechanisms of advective flow. Such an integration of physical and biological mechanisms within sandy sediment models was a major focus within two recent EU-supported research projects, termed COSA (COastal SAnds as biocatalytic filters; Huettel et al., 2006) and NAME (Nitrate from Aquifers and influences on the carbon cycling in Marine Ecosystems; Postma et al., 2005). For both biologically and physically induced pore water flows, the same three-step procedure is adopted, which has emerged over the years as the default strategy to model solute transport in complex natural environments (e.g. Elliott and Brooks, 1997a; Rena and Packman, 2004). [a] Process models are developed for a small-scale laboratory benchmark set-up, simulating the pore water flow and the resulting reactive transport. [b] In these simplified set-ups, tracer experiments are carried out under well-controlled conditions, and performance of the process model is validated against these data. [c] When the process model successfully reproduces the benchmark setup, it can be subsequently extrapolated to simulate flow patterns and tracer dynamics in more complex field conditions. For near-shore sandy sediments, the implementation of this (ambitious) scheme is in various stages of progress for the different mechanisms of pore water flow. Here, we report on the current state of model development, validation and application, reviewing past work as well as providing directions for future research. We show results from four different case studies: [1] lugworm bio-irrigation in laboratory set-up, [2] interaction of bio-irrigation and groundwater seepage on a tidal flat, [3] pore water flows in stirred benthic chambers, and [4] pore water flows induced by unidirectional flow over a sequence of ripples (see overview in Fig. 1). These case studies include both biologically and physically induced pore water flows, as well as simplified laboratory set-ups versus more complex field-like conditions.

\section{Model development: pore water flow and reactive transport}

The model formulation here targets a particular class of applications: the description of solute concentration patterns within the upper layer of sandy sediments and the quantification of the associated fluxes across the sediment-water 
interface. The model domain extends to $\sim 10-100 \mathrm{~cm}$ sediment depth, while the median particle size does not exceed $2 \mathrm{~mm}$ (thus excluding gravels). Four different case studies are examined relating to both biologically and physically induced flow. In these, we used the same software and we followed the same procedure to quantify flow patterns and tracer dynamics. This modeling approach consists of two parts. In a first step, the pressure and velocity fields within the pore water are described by a "flow model". In the development of this "flow model", the key aspects are the justification of the approximations in the momentum equation, the proper delineation of the model domain, and the selection of the appropriate boundary conditions at the boundaries of this model domain. In a second step, the computed velocity field is used as an input for a reactive transport model, which describes the evolution of certain tracer concentrations in the pore water. All model development and simulations are performed using finite element package COMSOL Multiphysics ${ }^{\mathrm{TM}}$ 3.2a. The scripts of the various models are freeware, and can be downloaded from http://www.nioo.knaw.nl/ppages/ogalaktionov/. These model scripts allow the user to adjust model domain geometry, as well as sediment and tracer properties.

\subsection{Simplification of the momentum equation}

The starting point for model development is the momentum balance for the pore water as derived in multi-phase continuum physics (e.g. Bear and Bachmat, 1991). At present, there is some debate on the level of detail that should incorporated in such a momentum balance, and as a result, existing models differ in their basic flow equation. Khalili et al. (1999) promoted the Darcy-Brinkman-Forchheimer (DBF) equation as a general equation to model pore flow

$$
\begin{aligned}
& \rho\left[\frac{1}{\phi} \frac{\partial \boldsymbol{v}_{d}}{\partial t}+\frac{1}{\phi}\left(\boldsymbol{v}_{d} \cdot \nabla\right) \boldsymbol{v}_{d}\right]= \\
& -\nabla p+\rho g \nabla z+\tilde{\mu} \nabla^{2} \boldsymbol{v}_{d}-\frac{\mu}{k} \boldsymbol{v}_{d}-\rho \frac{C_{f}}{\sqrt{k}}\left\|\boldsymbol{v}_{d}\right\| \boldsymbol{v}_{d}
\end{aligned}
$$

In this, $k$ denotes the permeability, $\mu$ the dynamic viscosity of the pore water, $\tilde{\mu}$ the effective viscosity, $\rho$ the pore water density, $C_{f}$ a dimensionless drag coefficient, $g$ the gravitational acceleration, and $z$ the vertical coordinate. The Darcy velocity $\boldsymbol{v}_{d}$ is related to the actual velocity of pore water as $\boldsymbol{v}_{d}=\phi \boldsymbol{v}$, where $\phi$ is the porosity. In the DBF equation, the total acceleration (left hand side) balances the forces due to pressure, gravitation, viscous shear stress (Brinkman), linear drag (Darcy), and non-linear drag (Forchheimer). The DBF Eq. (1) has the advantage of generality, but may also engender needless complexity. So the question is whether all terms in Eq. (1) are really needed for the applications that are targeted. As noted above, we exclusively focus on sands, that is, permeable sediments with a grain diameter less than $2 \mathrm{~mm}$. For sandy sediments, the DBF Eq. (1) can be substantially simplified. Numerical experiments by Khalili et al. (1997) showed that the acceleration and Forcheimer terms are negligibly small in sands. These two terms only become important when pore velocities are really high, like in highly-permeable gravel beds. Accordingly, the momentum balance for sandy sediments readily simplifies to

$$
-\nabla p+\rho g \nabla z+\tilde{\mu} \nabla^{2} \boldsymbol{v}_{d}-\frac{\mu}{k} \boldsymbol{v}_{d}=0
$$

This so-called Darcy-Brinkman equation forms the basis for the benthic chamber model of Khalili et al. (1997, 1999). However, one issue is whether the Brinkman term is really needed in the momentum equation. Khalili et al. (1999) argued that it should be included to accurately describe advective transport in sands. The Brinkman term is however only important in a transition layer between the overlying water and the sediment, where the velocity profile exhibits strong curvature $\left(\nabla^{2} \boldsymbol{v}_{d}\right.$ large). So, the question whether or not to retain the Brinkman term crucially depends on the thickness $\delta$ of this transition layer. Goharzadeh et al. (2005) investigated $\delta$-values for grain sizes from 1 to $7 \mathrm{~mm}$, and found that $\delta$ roughly equals the grain size diameter. In other words, in sands with a median grain size less than $2 \mathrm{~mm}$, the transition layer is small compared to the scale of the advective flow induced by waves, ripples and bio-irrigation, which is on the order of centimeters to decimeters. Accordingly, for practical modeling applications in sandy sediments on the scale of $10-100 \mathrm{~cm}$ depth, the Brinkman and other non-Darcian terms can be justifiably discarded. Note that this cannot be done for applications which specifically target processes within the uppermost surface layer (i.e. the model domain comprises the first few $\mathrm{mm}$ of the sediment). An important (and difficult) problem in this respect is the accurate coupling of the flow in the sediment to the free flow over the sediment. As our main focus is the inter-comparison of transport at depth (i.e. well below this surface layer), a detailed discussion of this boundary issue is beyond the scope of the present manuscript (see for example the theoretical analysis in Zhou and Mendoza, 1993).

\subsection{Flow model}

From the viewpoint of the overlying water, the neglect of the small Brinkman transition layer implies that the sediment is regarded as a solid body, and a no-slip boundary condition applies at the sediment-water interface. From the viewpoint of the sediment, it implies that the momentum balance (1) simply reduces to Darcy's law

$\boldsymbol{v}_{d}=-\frac{k}{\mu}(\nabla p-\rho g \nabla z)$

Equation (3) is the standard formulation in models of sandy sediment advection (Savant et al., 1987; Brooks et al., 1997a; Meysman et al., 2005; Rena and Packman, 2004; Cardenas and Wilson, 2007), and is also the starting point of all model development here. We assume that the pore water is 
incompressible and that the tracer concentration does not affect the density $\rho$. Then, introducing the effective pressure $p_{e}=p-\rho g z$ as the excess pressure over the hydrostatic pressure, one can substitute Darcy's law (3) into the continuity equation $\nabla \cdot \boldsymbol{v}_{d}=0$, to finally obtain

$\nabla \cdot\left(-\frac{k}{\eta} \nabla p_{e}\right)=0$

We further assume that the sediment is homogeneous, and so the permeability, porosity, and pore water viscosity remain uniform over the model domain (see Salehin et al., 2004 for a more complex model formulation in heterogeneous sediments). Under the condition of homogeneity, expression (4) reduces to the classical Laplace equation

$$
\nabla^{2} p_{e}=0
$$

Appropriate boundary conditions are needed to compute the pressure distribution and the associated pore water velocity field from Eqs. (5) and (3). These boundary conditions differ between individual models and are discussed below.

\subsection{Reactive transport model}

Once the pore water velocity $v$ is computed, it can be substituted in the mass conservation equation for a solute tracer in the pore water (Bear and Bachmat, 1991; Boudreau, 1997; Meysman et al., 2006)

$\frac{\partial C_{s}}{\partial t}+\nabla \cdot\left(-\boldsymbol{D} \cdot \nabla C_{s}+v C_{s}\right)-R=0$

where $C_{s}$ denotes the tracer concentration, $\boldsymbol{D}$ denotes the hydrodynamic dispersion tensor and $R$ represents the overall production rate due to chemical reactions. Note that $R$ can be spatially variable, and that consumption processes are accounted for as negative production. The hydrodynamic dispersion tensor can be decomposed as $\boldsymbol{D}=\boldsymbol{D}^{\mathrm{mol}}+\boldsymbol{D}^{\text {mech }}$, representing the effects of both molecular diffusion and mechanical dispersion (Oelkers, 1996). The part due to molecular diffusion is written as (Meysman et al., 2006)

$\boldsymbol{D}^{\mathrm{mol}}=(1-2 \ln \phi)^{-1} D^{\mathrm{mol}} \boldsymbol{I}$

where $D^{\mathrm{mol}}$ is the molecular diffusion coefficient, $\boldsymbol{I}$ is the unit tensor, and the multiplier $(1-2 \ln \phi)^{-1}$ forms the correction for tortuosity (Boudreau, 1996). The mechanical dispersion tensor $\boldsymbol{D}^{\text {mech }}$ is given the classical form (Freeze and Cherry, 1979; Lichtner, 1996)

$\boldsymbol{D}^{\mathrm{mech}}=D_{T} \boldsymbol{I}+\frac{1}{\|\boldsymbol{v}\|^{2}}\left(D_{L}-D_{T}\right) \boldsymbol{v} \boldsymbol{v}^{T}$

The quantities $D_{T}$ and $D_{L}$ are the transversal (perpendicular to the flow) and longitudinal (in the direction of the flow) dispersion coefficients, which are expressed as functions of grain-scale Peclet number $P e \equiv d_{p}\|\boldsymbol{v}\| / D^{\mathrm{mol}}$ where $d_{p}$ stands for the median particle size (Oelkers, 1996).

$D_{T}=D^{\mathrm{mol}} 0.5\{P e\}^{1.2}$
$D_{L}=D^{\mathrm{mol}} 0.015\{P e\}^{1.1}$

The Formulas (9) and (10) are valid for Peclet numbers in the range 1-100, which applies to the models presented here (this was verified a posteriori).

\subsection{Implementation and numerical solution}

In the simulations presented here, we assume that environmental parameters (porosity, permeability, diffusion coefficients) do not vary over the model domain, primarily to facilitate the comparison between models. Spatial heterogeneity can be important under natural conditions (e.g. the permeability in a sand ripple can be markedly different from the sediment below), yet its importance for flow patterns and tracer exchange has only been addressed in a few pioneering studies (Salehin et al., 2004), and hence, this is clearly a topic for future research. Furthermore, we assume that changes in the tracer concentration do not significantly influence the pore water properties (density, viscosity). In most cases, this is a realistic assumption that significantly simplifies the simulations, as the flow model becomes decoupled from the reactive transport model. Accordingly, the flow model is solved first, and subsequently, the resulting flow field is imported into the reactive transport model. The flow model is implemented using the "Darcy's Law" mode in the Chemical Engineering Module of COMSOL Multiphysics 3.2a. The reactive transport model is implemented using the "Convection and Diffusion" mode of the same Chemical Engineering Module. All model parameters can be adjusted in corresponding script files (M-files). These files can be executed via the MATLAB interface of COMSOL, or they can be run using the newly developed COMSOL Scripts ${ }^{\mathrm{TM}}$ package. The usage of scripting allows an easy adjustment of model constants and geometrical parameters.

\section{Description of four case studies}

We now implement the modelling approach detailed in the previous section to describe flow and tracer dynamics in four different case studies. Each of these four models applies the same set of model equations: the Laplace Eq. (5) for the flow and the reactive-transport Eq. (6) for the tracer dynamics. Moreover, each of the four models incorporates a similar model domain: either the isolated sediment core that is used in laboratory incubations, or some representative sediment compartment in the field. In all cases, the lower and lateral sides of the model domain are considered as closed. Therefore, the principal difference between the four models becomes the interaction between the overlying water and the sediment: the models will differ in the geometry of the sediment-water interface and the boundary conditions that are specified along this interface. 

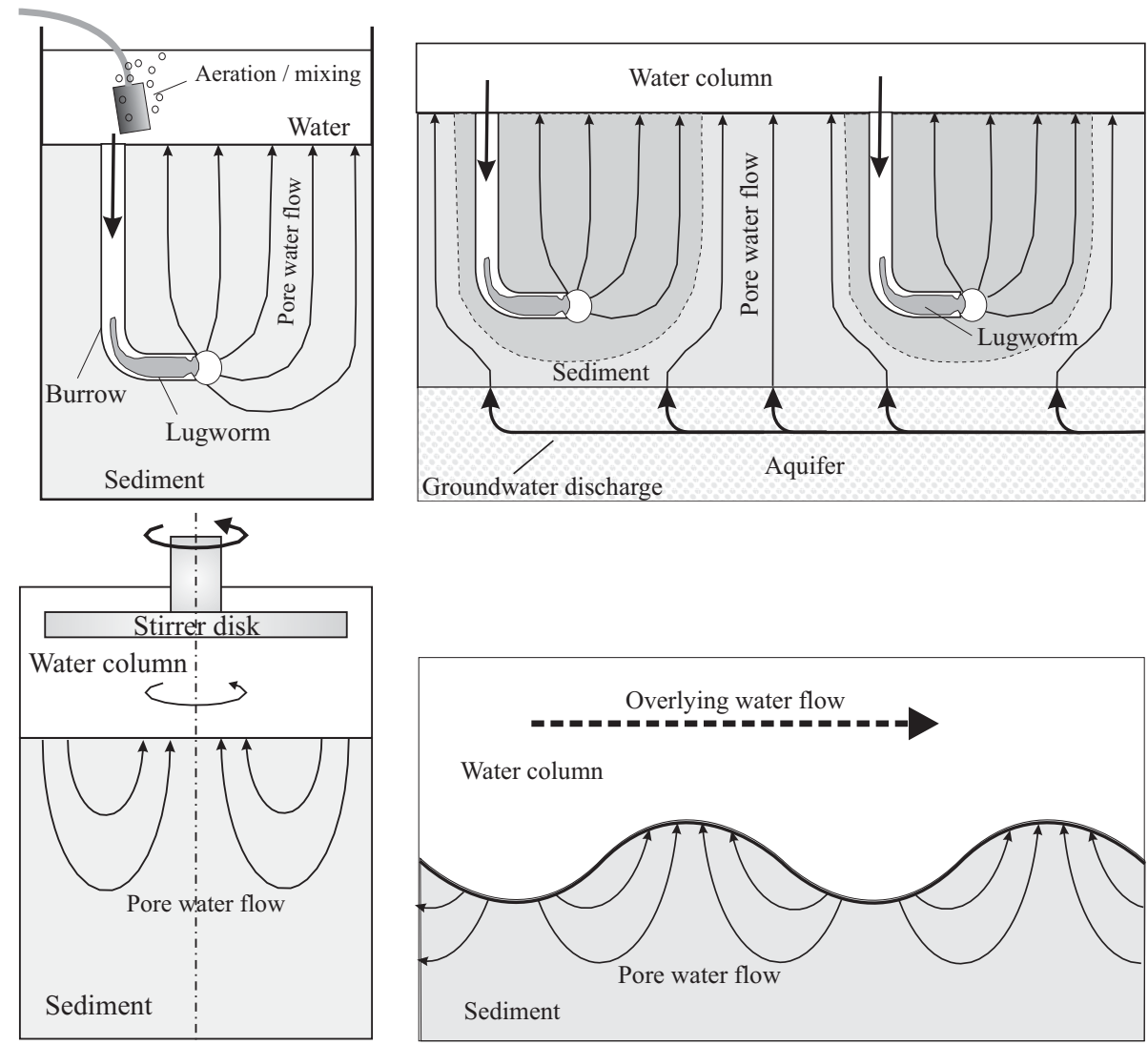

Fig. 1. Schematic representation of the four situations that are modeled: (a) Laboratory incubation set-up commonly used to study lugworm bio-irrigation; (b) Lugworm bio-irrigation interfering with groundwater seepage on a tidal flat; (c) Pore water flow in stirred incubation chamber; (d) Unidirectional flow over a ripple field inducing pore water flow.

\subsection{Bio-irrigation in a laboratory set-up: model [1]}

Our case study of biological flow focuses on the advective bio-irrigation induced by the lugworm Arenicola marina. Lugworms are abundant in coastal sandy areas worldwide, and are dominant bio-irrigators in these systems (Riisgård and Banta, 1998). They inject burrow water at depth, which causes an upward percolation of pore water and seepage across the sediment-water interface (Timmermann et al., 2002; Meysman et al., 2005). Because of their strong irrigational effects, lugworms are frequently used in studies of advective bio-irrigation (Foster-Smith, 1978; Riisgård et al., 1996). Model [1] describes the laboratory incubation set-up (Fig. 1a) that is commonly used to study lugworm bio-irrigation. Model [1] was proposed in Meysman et al. (2006a) to analyze lugworm incubation experiments with inert tracers. In a follow-up study on model complexity, model [1] was compared to a range of simpler and more complex bio-irrigation models (Meysman et al., 2006b).

\subsubsection{Problem outline}

Lugworms pump overlying water into their J-shaped burrows, which is then discharged into the sediment, across the walls of a so-called "injection pocket" at the end of the burrow. The burrow walls essentially form an extension of the sediment-water interface. So when modeling lugworm bioirrigation, a key issue is the representation of the internal structure of the lugworm burrow. This problem was recently investigated in detail by Meysman et al. (2006b), where 3$\mathrm{D}$ models of the J-shaped burrow and the surrounding sediment were compared to simplified 2-D and 1-D versions. The basic conclusion was that to a good approximation, the actual geometry of the burrow can be ignored when modeling the pore water flow. This is because the walls of the burrow are impermeable to flow, with the exception of the injection pocket. The burrow thus acts as an impenetrable obstacle to the flow, and because the burrow is small, this only causes a small distortion of the flow pattern. So, it suffices to model the injection pocket where the burrow water is actually pumped into the surrounding sediment (Meysman et al., 2006a, b). Adopting a spherical (radial-symmetric) injection 


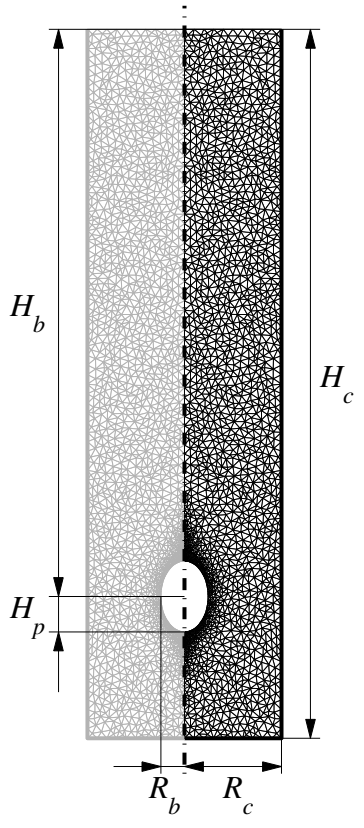

b

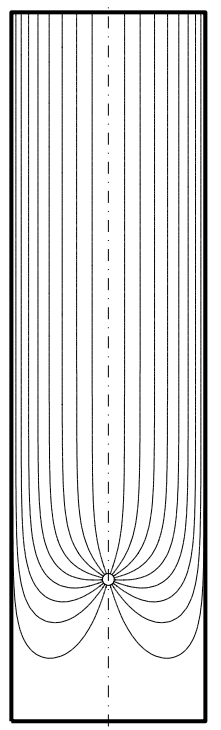

$\mathrm{c}$

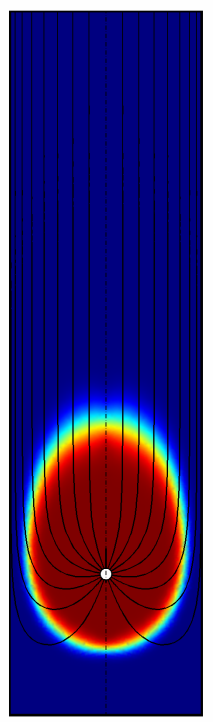

d

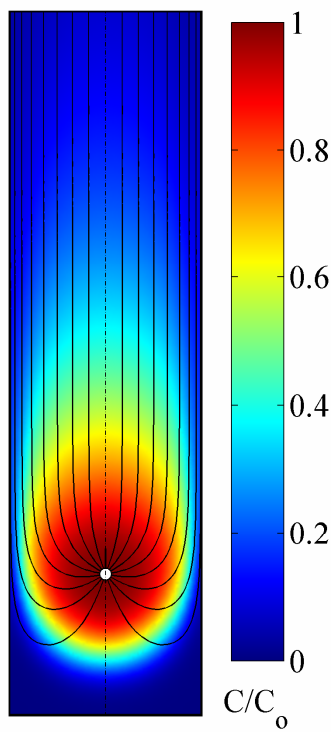

Fig. 2. Model [1]: lugworm bio-irrigation in a laboratory core set-up. (a) scheme of the sediment domain geometry and the finite element mesh; (b) simulated flow line pattern; (c) simulated concentration pattern for the conservative tracer bromide after two hours of flushing; (d) steady-state concentration pattern of oxygen. Concentrations are expressed relative to the constant concentration in the overlying water column.

pocket, one can introduce a substantial model simplification (Fig. 2a). Physically, the model domain remains 3-D, but mathematically, it can be described by a radial-symmetric 2$\mathrm{D}$ model that incorporates two variables (the depth $z$ and the distance $r$ from the central symmetry axis).

\subsubsection{Model domain and boundary conditions}

The sediment domain is a cylinder of radius $R_{S}$ and height $H_{s}$. The injection pocket is represented as a spherical void of radius $R_{b}$ located at the domain's symmetry axis at depth $H_{b}$. A triangular unstructured mesh was used to discretize the model domain with a higher resolution near the injection pocket (Fig. 2a). At the lateral sides and the bottom of the incubation core the no-flow condition is adopted. The actual sediment-water interface consists of two parts: the flat sediment surface and the surface of the injection pocket. At the sediment surface, the excess pressure is constant and set to zero, which allows the pore water to leave the sediment domain. Along the surface of the injection pocket the lugworm's pumping activity imposes a constant excess pressure $p_{e}^{i p}$. However, no data are available on $p_{e}^{i p}$ as this parameter is difficult to access experimentally. Instead, the lugworm's pumping rate $Q$ is usually reported, which must match the discharge of water across the surface of the injection pocket. When the size of the injection pocket is small compared to both the burrow depth $H_{b}$ and the sediment core radius $R_{s}$, one can directly impose a uniform flux $\left\|\boldsymbol{v}_{d}\right\|=Q / A_{i p}$ along the injection pocket, where $A_{i p}$ is the surface area. When the injection pocket is small, pressure variations along its surface will be small, and so the flow field that is generated by imposing a uniform flux will be identical as when imposing a constant pressure $p_{e}^{i p}$. When the size of the injection pocket is relatively large, we implemented a two-step procedure to derive the required $p_{e}^{f p}$ value from the known pumping rate $Q$. First, the flow field is calculated for some trial value $p_{e}^{\text {test }}$ and the associated discharge $Q^{\text {test }}$ is evaluated. Then due to the linearity of Darcy's law, the pressure $p_{e}^{i p}$ corresponding to the actual flux $Q$ is simply calculated as

$p_{e}^{i p}=p_{e}^{\text {test }} \frac{Q}{Q^{\text {test }}}$

Both types of boundary conditions options (small and large injection pockets) are implemented as alternative options in the M-file, which contains the model script.

\subsection{Interaction between bio-irrigation and upwelling} groundwater in an intertidal sediment: model [2]

Model [2] basically implements the same bio-irrigation process as model [1], but now in a field application for a Danish beach site, where the bio-irrigation of lugworms interacts with the upwelling of fresh groundwater (Fig. 1b). 
a

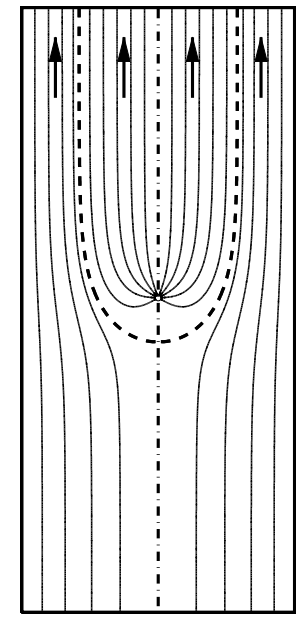

b

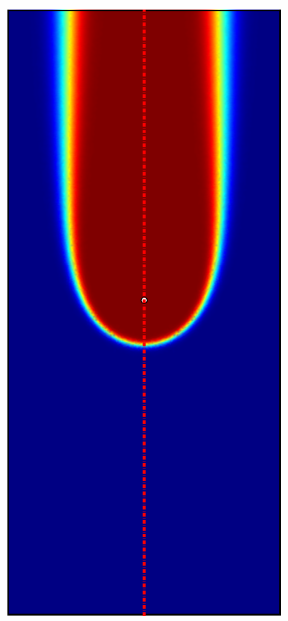

$\mathrm{c}$

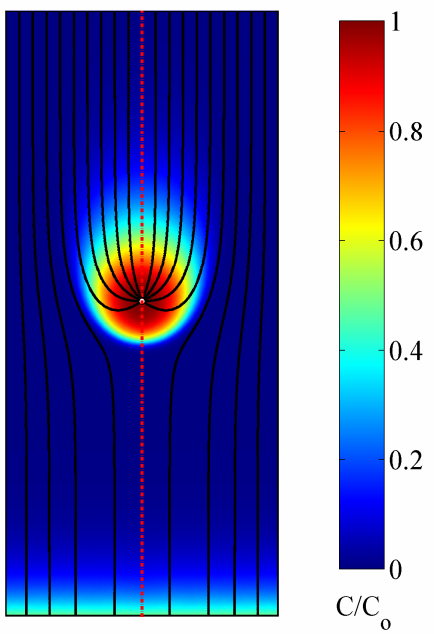

Fig. 3. Model [2]: interaction between lugworm bio-irrigation and upwelling groundwater on a tidal flat. (a) Sediment domain geometry and flow line pattern. The bold dashed line separates marine conditions (injection of overlying sea water by the lugworm) and fresh conditions (ground water upwelling from below); (b) simulated concentration pattern for the conservative tracer bromide at steady state; (c) steady-state concentration pattern of oxygen. Concentrations are expressed relative to the constant concentration in the overlying water column.

\subsubsection{Problem outline}

In recent decades, there is an increased transfer of nitrate from terrestrial areas with intense agriculture to the coastal zone. This results in the eutrophication of coastal waters, causing algal blooms and deterioration of water quality, with significant effects on biodiversity and ecosystem functioning. The default assumption is that most nitrate reaches the sea via riverine input, yet an unknown quantity percolates through the seabed as nitrate-rich groundwater. To this end, the discharge of nitrate-bearing groundwater into Ho Bay (Wadden Sea, Denmark) was investigated within the multi-disciplinary EU-project NAME (Postma, 2005). The tidal fluctuation in Ho Bay is about $1.5 \mathrm{~m}$, and ground water (nitrate $\sim 0.6 \mathrm{mM}$, no sulfate) percolates up through the sandy beach at localized sites within the intertidal area (nitrate $\sim 0.03 \mathrm{mM}$, sulfate $20 \mathrm{mM}$ ).

Within the upper centimeters of the sediment, the groundwater-derived nitrate comes into contact with fresh organic matter, originating from primary production within the bay. Because of the high nitrate concentrations, one would expect that denitrification would be the dominant pathway of organic matter mineralization within the surface sediment. Although high denitrification rates were measured, an intriguing observation was that the sediment also contained high pyrite concentration, thus indicating sulfate reduction (G. Lavik, personal communication). This would imply that two parallel pathways of organic matter processing are active: a terrestrial (using nitrate from ground water) along a marine (using sulfate from the overlying seawater). Based on thermodynamic free-energy constraints and bacterial physiology, denitrification and sulfate reduction are mutually exclusive, and therefore, a proper explanation was needed to reconcile the occurrence of these two parallel pathways.

One hypothesis that was forwarded as a possible explanation focused on the interaction between lugworm bioirrigation and the upwelling of groundwater. Lugworms are the dominant macrofauna species in the Ho Bay intertidal area where groundwater seepage occurred, with densities up to 25 ind. $\mathrm{m}^{-2}$. The injection of overlying seawater by these lugworms could create marine micro-zones within an otherwise freshwater dominated sediment (Fig. 1b). To investigate this effect, model [2] was newly developed (in contrast to model [1], model [2] has not been previously reported in literature).

\subsubsection{Model domain and boundary conditions}

Similar to model [1] we consider a cylindrical sediment domain (Fig. 3a). Rather than a laboratory core, this model domain now represents the territory of a single lugworm on the Ho Bay field site. The domain radius can be calculated as $R_{S}=(N \pi)^{-\frac{1}{2}}$, where $N$ is the number of lugworms per unit area. Using the maximal observed value of $25 \mathrm{ind} . \mathrm{m}^{-2}$, the radius of an average lugworm territory thus becomes $11.3 \mathrm{~cm}$. The geometry of the injection pocket (radius $0.25 \mathrm{~cm}$ and depth $24 \mathrm{~cm}$ ) and the pumping rate $\left(1 \mathrm{~cm}^{3} \mathrm{~min}^{-1}\right)$ are those of a "standard" adult lugworm (Meysman et al., 2006a). The difference with model [1] is the upward pore water flux that is prescribed at the lower 
boundary of the model domain (depth $50 \mathrm{~cm}$ ). The interaction of this upward flow with the lugworm's irrigation leads to a different flow pattern. An upward velocity of $0.3 \mathrm{~cm}$ $\mathrm{h}^{-1}$ was specified at the lower boundary, based on field observations. As a result, the groundwater flow from below $\left(120 \mathrm{~cm}^{3} \mathrm{~h}^{-1}\right)$ is two times larger than the lugworm's pumping rate $\left(60 \mathrm{~cm}^{3} \mathrm{~h}^{-1}\right)$.

\subsection{Pore water flow in a benthic chamber: model [3]}

In our case study of physically induced flow, we focus on the consequences of unidirectional currents over ripple fields. To get a better control on experimental conditions, Huettel and Gust (1992a) proposed a benthic chamber set-up that mimics the pressure variations induced by flow over a sand ripple. A rotating disc creates a circular movement in the overlying water, and as a result of this rotational flow, radial pressure gradients develop that lead to an advective exchange between pore water and overlying water (Fig. 1c). These benthic chambers are now frequently used to study the biogeochemical effects of sediment-water exchange in sandy sediments (Glud et al., 1996; Janssen et al., 2005b; Billlerbeck et al., 2006). Model [3] describes the pore water flow and tracer dynamics induced within these benthic chambers. It was proposed by Cook et al. (2006) to quantify the influence of advection on nitrogen cycling in permeable sediments. Related chamber models were presented in Khalili et al. (1997) and Basu and Khalili (1999), though starting from a more complex equation set (see discussion in Sect. 2)

\subsubsection{Problem outline}

Physically-induced pore water flow originates from pressure gradients at the sediment surface. Accordingly, the critical issue in model development is the representation of the pressure distribution at the sediment-water interface. This applies to the benthic chamber system, but also to other types of physically-induced flow (ripples, waves). There are two possible approaches. One approach depends entirely on modeling: one derives the sought-after pressure distribution from a coupled hydrodynamic model of the water column (e.g. Cardenas and Wilson, 2007). In the benthic chamber, this implies that we explicitly model the rotational flow in the overlying water starting from the basic Navier-Stokes equations of hydrodynamics (Khalili et al., 1997; Basu and Khalili, 1999). The advantage of the modeling approach is that it can always be implemented. An important drawback is that it requires a proper parameterization of a complex flow model in a complex geometry, and when this is not the case, the simulated results may be far from reality. The alternative approach is to rely on experiments and derive an empirical pressure distribution at the sediment surface. In such experiments, the sediment surface is replaced by solid wall, and small pressure gauges are inserted (Huettel and Gust, 1992a; Huettel et al., 1996; Janssen et al., 2005a). The resulting expression for the pressure distribution along the sedimentwater interface can be then used as a forcing function for a sediment model. The experimental approach avoids the application of a complex flow model, but usually such data are simply not available. For the benthic chamber system, the required pressure distributions have been measured (Huettel and Gust, 1992b; Janssen et al., 2005a). Therefore, in model [3], we can follow the experimental approach. In model [4], we will illustrate the modeling approach.

\subsubsection{Model domain and boundary conditions}

Mimicking the benthic chamber, the model domain consists again of a cylindrical sediment core of radius $R_{S}$ and height $H_{s}$ (Fig. 4a). We assume that the sediment-water interface is perfectly flat, and that a perfectly radial-symmetric stirring pattern establishes in the overlying water. In this scenario, the pressure distribution at the sediment surface will be radial-symmetric, and so, the pore water flow and concentration patterns within the sediment will show the same symmetry. Just like in bio-irrigation model [1], the model domain thus receives radial-symmetric 2-D description that incorporates two variables (the depth $z$ and the distance $r$ from the central symmetry axis). A triangular unstructured mesh was used to discretize the model domain, with an element size of $\sim 0.1 \mathrm{~mm}$ at the sediment surface, increasing to $2 \mathrm{~mm}$ at the lower boundary of the core (Fig. 4a). Along the bottom and the lateral walls, we implemented a no-flux condition. Following Cook et al. (2006), we adopted the following pressure relation at the sediment surface

$p(r, 0)=2 p_{0}(\omega)\left(\frac{r}{R_{S}}\right)^{2}\left(1-\frac{1}{2}\left(\frac{r}{R_{S}}\right)^{2}\right)$

where $p_{0}(\omega)$ is the pressure difference between the outer edge and the center of the chamber. The value of $p_{0}(\omega)$ depends on the rotation frequency $\omega$ of the stirrer disk, and was measured to be $3.5 \mathrm{~Pa}$ at $40 \mathrm{rpm}$ and 14.4 at $80 \mathrm{rpm}$ (Billerbeck et al., unpublished results).

\subsection{Pore water flow induced in a ripple bed: model [4]}

Model [3] simulates the benthic chamber, which in itself was introduced as a physical analogue that mimics pore water flow under sand ripples. In contrast, model [4] directly simulates the free flow over the sand ripples that causes pressure variations at the interface with the overlying water, which in turn, induces pore water flow beneath the ripples.

\subsubsection{Problem outline}

Unidirectional flow over sand ripples has been experimentally studied in flume tanks for configurations consisting of a single symmetric ripple (e.g. Huettel et al., 1996) as well as regularly spaced asymmetric sand ripples (e.g. Thibodeaux and Boyle, 1987; Savant et al., 1987; Elliott and Brooks, 


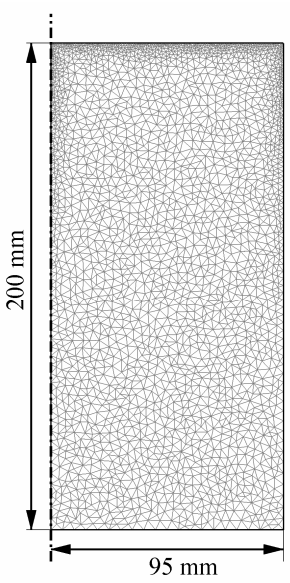

b

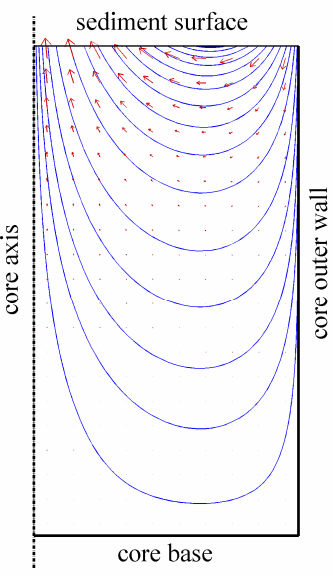

C

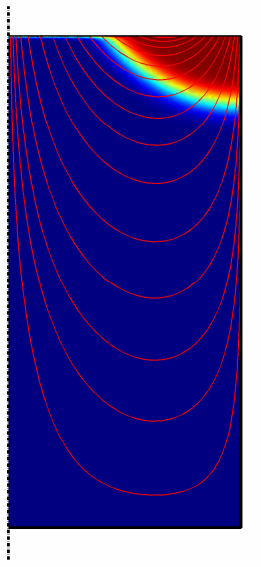

d

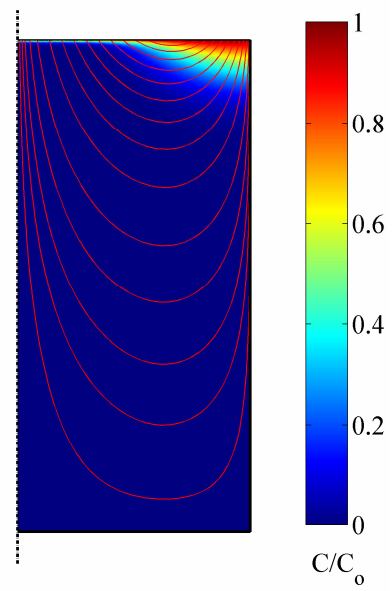

Fig. 4. Model [3]: pore water flow induced in a stirred benthic chamber. (a) geometry of the sediment domain and the finite element discretization; (b) flow line pattern; (c) bromide concentration pattern after $12 \mathrm{~h}$ of incubation with the rotation speed of the stirrer disk set to $40 \mathrm{rpm}$; (d) steady-state oxygen distribution (at $40 \mathrm{rpm}$ ). Concentrations are expressed relative to the constant concentration in the overlying water column.

1997b; Rena and Packman, 2004, 2005). As was discussed for the benthic chamber, the critical component of the model is again the pressure distribution at the ripple surface. As noted earlier, such pressure relations can be obtained from either modeling or experiments. In model [3], we implemented the latter option, and this is also the approach taken in previous models of ripple-induced flow (Savant et al., 1987; Elliott and Brooks, 1997a; Rena and Packman, 2004, 2005). Here, in model [4], the pressure relation at the ripple surface is obtained by modeling the interaction between the overlying water flow and the ripple using the Navier-Stokes equations of hydrodynamics. A crucial choice in this is whether to represent the flow regime in the overlying water as laminar or turbulent. Until now, studies on advective exchange have either implicitly or explicitly assumed that the flow regime remains laminar (Khalili et al., 1997; Basu and Khalili, 1999; Cardenas and Wilson, 2007). However, when calculating a characteristic Reynolds number under field conditions, using a characteristic range of flow velocities $\left(u=10-100 \mathrm{~cm} \mathrm{~s}^{-1}\right)$ and ripple heights $\left(H_{r}=1-10 \mathrm{~cm}\right)$, one finds relatively high Reynolds numbers $R e=\left(\rho u H_{r}\right) / \mu$ in the range from $10^{3}$ to $10^{5}$. Although the exact transition from laminar to turbulent depends on local flow conditions, it usually commences around Reynolds numbers between $5 \times 10^{3}$ and $10^{4}$. Accordingly, under field conditions, a combination of low flow over small ripples may still lead to laminar flow, but high flow velocities over relatively large ripples could definitely generate turbulent conditions. Laminar and turbulent descriptions of the overlying flow differ considerably. Until now, it has not been assessed how the laminar vs. turbulent description of the water column affects the pore water flow and tracer dynamics in the sediment. To test this, we performed exactly the same simulations with laminar and turbulent models. The laminar flow model is similar to the ripple models of Cardenas and Wilson (2006, 2007), but for some minor details (rounded ripple geometry; implementation of the periodicity of the model domain).

\subsubsection{Water column model}

The water column has a height $H_{w}=40 \mathrm{~cm}$, and the direction of the flow is in the direction of the $\mathrm{x}$-axis (from left to right in Figs. 5a and 6a). The upper boundary of the water column is flat (no waves). The lower boundary displays the topography of the sediment-water interface, and consists of a flat bottom section followed by a sequence of identical ripples. The flat section is introduced to allow the development of a representative vertical velocity profile, before the flow hits the first ripple. The subsequent ripple sequence is relatively long, so that the flow pattern in the overlying water column is well-established and does not change significantly from ripple to ripple. The number of ripples specified by the input parameter $n_{r}$, which in the present examples is set to 10. Each ripple has the shape of an asymmetric triangle with a gentle front slope and steeper back slope. The shape of the ripples is specified by four parameters, which all can be adjusted. The default values are the following: the ripple length is set to $L_{r}=20 \mathrm{~cm}$, the ripple height is set to $H_{r}=2.13 \mathrm{~cm}$, and the position of the ripple crest is at $L_{\max }=15.67 \mathrm{~cm}$. The crests and troughs are rounded: the curvature radius of these rounded corners is set to $r_{c}=3 \mathrm{~cm}$.

The flow settings were those of moderate flow $\left(10 \mathrm{~cm} \mathrm{~s}^{-1}\right)$ 
over a relatively small ripple $(\sim 2 \mathrm{~cm})$, resulting in a Reynolds number around 2000. Such a Reynolds number lies in the transition region from laminar to turbulent flow. Under natural conditions, it would most likely lead to turbulent flow. The laminar simulation would not apply then, but the results are still useful for comparison purposes. Laminar and turbulent flow regimes are implemented using the "Incompressible Navier-Stokes" and " $k-\varepsilon$ Turbulence Model" modules respectively from the Chemical Engineering Module of Comsol Multiphysics 3.2a. Both models have similar boundary conditions at the upper and right boundary of the model domain. In both laminar and turbulent regimes, the slip/symmetry boundary condition is specified at the upper boundary (mimicking a free surface). Outflow conditions (zero excess pressure) are implemented at the outflow boundary on the right. At the inflow and at the sedimentwater interface, different boundary conditions are implemented in both laminar and turbulent regimes. In the laminar flow model, the power-profile $u(y)=u_{\max }\left(y / H_{w}\right)^{0.14}$ is prescribed at the inflow where $u_{\max }=10.3 \mathrm{~cm} \mathrm{~s}^{-1}$. This prescribed profile mimics a fully developed boundary layer, thus reducing transient effects along the ripple sequence. A noslip boundary condition is prescribed at the sediment - water interface. For the turbulent flow regime, a plug velocity profile is specified at the inflow on the left (constant horizontal velocity $u=u_{\max }=10 \mathrm{~cm} \mathrm{~s}^{-1}$ ). When the flow is established after a few ripples, the horizontal velocity at the upper boundary of the water column becomes $10.7 \mathrm{~cm} \mathrm{~s}^{-1}$. At the sediment-water interface, we implemented the "logarithmic law of the wall" condition available in $k-\varepsilon$ module.

\subsubsection{Pore water flow model}

The sediment domain is a rectangular two-dimensional "box" of width $L_{r}=20 \mathrm{~cm}$ and depth $H_{s}=20 \mathrm{~cm}$. It is placed under a single ripple spanning the distance between two consecutive ripple troughs. To reduce the (artificial) influence of the outflow section, the sediment "box" is placed under the 7-th ripple (out of 10 ripples in total). The position of the "test" ripple can be adjusted by modifying the appropriate parameter $m_{\text {ripple }}$ in the script. The side and bottom walls of this "sediment box" are set impenetrable to flow.

\subsubsection{Numerical issues}

The equation system of the $\mathrm{k}-\varepsilon$ turbulence model is strongly non-linear, and its solution consumes far more memory than the laminar flow solution. We encountered problems when solving the k- $\varepsilon$ turbulence model for a moderate mesh resolution. The simulation did not lead to convergence with actual parameter values (actual viscosity and density of sea water) and zero-velocity initial conditions. To get around the problem, we used an iterative procedure, where we first artificially increased the dynamic viscosity of the overlying water a 1000-fold. This solution was then used as initial condition for a simulation with 10 time lower dynamic viscosity. After four iterations, the desired solution was obtained for the true value of the viscosity.

\section{Model validation: simulation of inert tracer experi- ments}

\subsection{Tracer experiments as model validation}

In order to arrive at true quantification, the underlying flow and tracer models should be properly validated against experimental data. Unfortunately, a fundamental drawback is that it is hard to directly measure the basic hydrodynamic variables that govern the flow pattern (pressure and pore velocity). Although piezoelectric pressure sensors can be placed in the sediment (Massel et al., 2004), these sensors are too large to resolve the $\mathrm{cm}$-scale pressure gradients involved in bio-irrigational or ripple-induced advection. Moreover, such sensors only provide one-dimensional point measurements, while in reality the flow pattern is three-dimensional. To fully capture the geometry of flow pattern, one thus would require a high-resolution array of point measurements at the same resolution as the model. Recently, Cardenas and Wilson (2007) did just that for laminar flow over ripples. They compared simulations to experimental data for flow, turbulence parameters and most importantly, the pressure along the interface of the ripple, which closely fitted the experimental data of Elliott and Brooks (1997b).

Lacking direct measurements, researchers have turned to (indirect) tracer methods to visualize the interstitial pore water flow. Different approaches have been implemented: (1) adding tracer to the overlying water column and examining the tracer distribution in the pore water through sampling ports (e.g. Elliott and Brooks, 1997b), transparant flume walls (e.g. Salehin et al., 2004; Rena and Packman, 2005) or core sectioning (e.g. Rasmussen et al., 1998), (2) saturating the pore water with tracer, and measuring the appearance of the tracer in the overlying water (Huettel and Gust, 1992a; Meysman et al., 2006a), (3) adding dyes to localized zones of pore water (spots or layers), and visualizing the migration and dispersion of these dyed patterns (Huettel and Gust, 1992a; Precht and Huettel, 2004; Salehin et al., 2004), and (4) adding tracer to the overlying water, and measuring the decrease of the tracer in the overlying water (e.g. Glud et al., 1996; Salehin et al., 2004).

Until now, tracer experiments have been mostly used in a semi-quantitative way (1) to visualize the flow pattern within the sediment, (2) to estimate flow velocities at specific points by tracking the progression in time of a dye front, and (3) to calculate the overall exchange between sediment and overlying water. Relatively few studies have compared tracer data with the output of corresponding tracer models (Khalili et al., 1997; Rena and Packman, 2004, 2005; Salehin et al., 2004; Meysman et al, 2006a). Such a "thorough" validation 


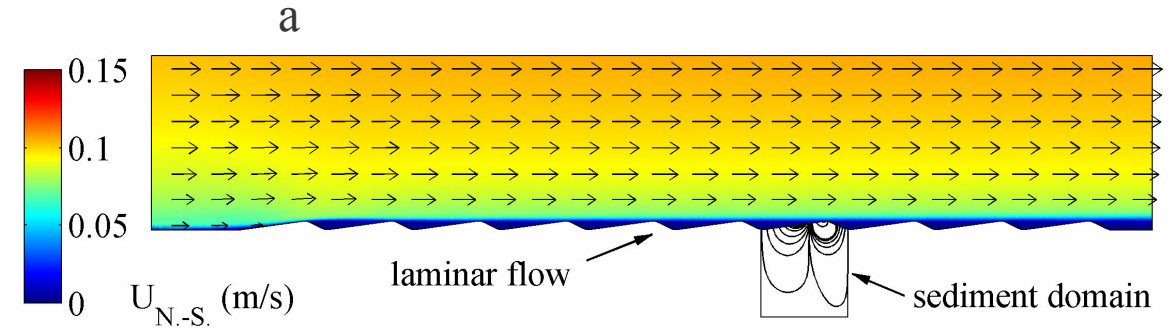

b

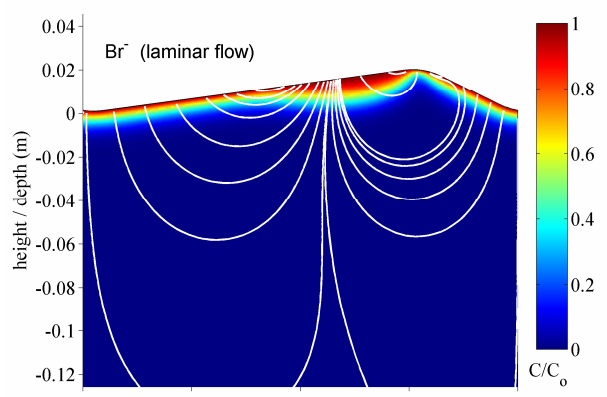

$\mathrm{C}$

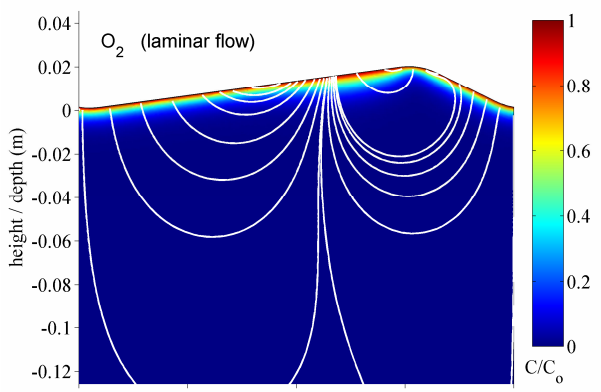

Fig. 5. Model [4], laminar variant: Unidirectional flow over a ripple field with laminar flow conditions. (a) velocity field in the water column; (b) flow line pattern and bromide distribution after $12 \mathrm{~h}$; (c) flow line pattern and steady-state oxygen distribution. Concentrations are expressed relative to the constant concentration in the overlying water column.

$\mathrm{a}$

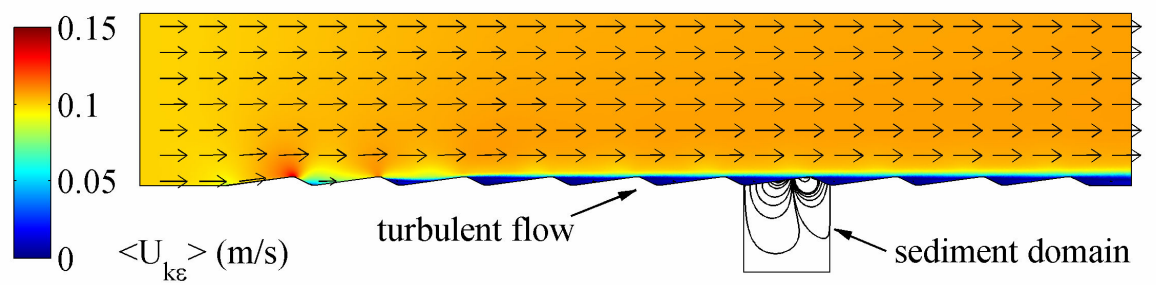

b

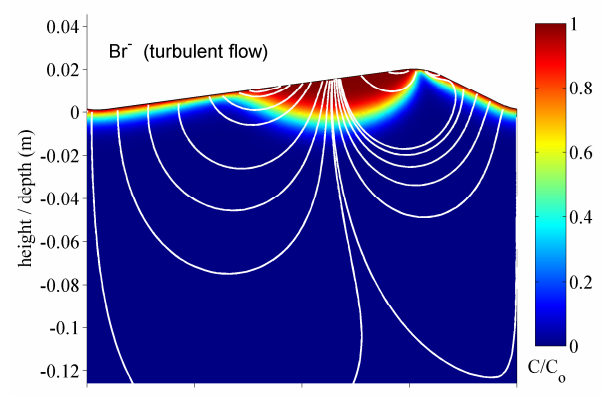

$\mathrm{C}$

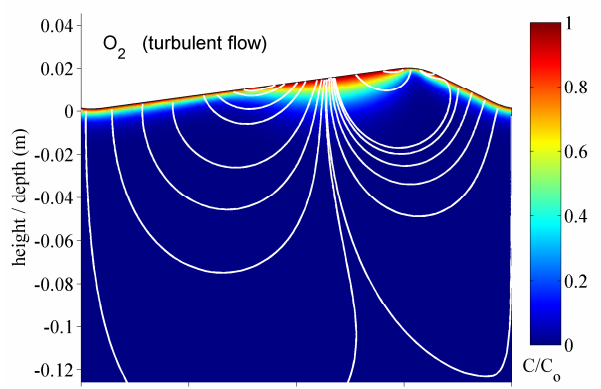

Fig. 6. Model [4], turbulent variant: Unidirectional flow over a ripple field with turbulent flow conditions. (a) velocity field in the water column; (b) flow line pattern and the bromide distribution after $12 \mathrm{~h}$; (c) flow line pattern and steady-state oxygen distribution. Concentrations are expressed relative to the constant concentration in the overlying water column. 
Table 1. Parameter values used in the simulations of lugworm bio-irrigation.

\begin{tabular}{|c|c|c|c|c|c|}
\hline Core set-up & & & Run1 & Run2 & $\mathrm{Br}^{-} / \mathrm{O}_{2}$ \\
\hline Core radius & $R_{S}$ & $\mathrm{~cm}$ & 5.0 & 5.0 & 5 \\
\hline Sediment height & $H_{S}$ & $\mathrm{~cm}$ & 9.6 & 9.4 & 30 \\
\hline Height overlying water column & $H_{w}$ & $\mathrm{~cm}$ & 2.6 & 2.9 & NA \\
\hline \multicolumn{6}{|l|}{ Sediment parameters } \\
\hline Temperature & $T$ & ${ }^{\circ} \mathrm{C}$ & 20 & 20 & 15 \\
\hline Salinity & $S$ & - & 30 & 30 & 30 \\
\hline Porosity & $\phi$ & - & 0.38 & 0.38 & 0.5 \\
\hline Permeability & $\mathrm{c}$ & $\mathrm{m}^{2}$ & $1.0 \times 10^{-11}$ & $1.0 \times 10^{-11}$ & $1.0 \times 10^{-11}$ \\
\hline Median grain size & $\bar{r}$ & $\mu \mathrm{m}$ & 263 & 263 & 220 \\
\hline \multicolumn{6}{|l|}{ Bio-irrigation parameters } \\
\hline Depth injection pocket & $H_{i p}$ & $\mathrm{~cm}$ & 4.45 & 9.05 & 24 \\
\hline Radius injection pocket & $R_{i p}$ & $\mathrm{~cm}$ & 0.2 & 0.2 & 0.25 \\
\hline Pumping rate & $Q$ & $\mathrm{~cm}^{3} \min ^{-1}$ & 0.35 & 1.0 & 1 \\
\hline
\end{tabular}

of flow and tracer models is definitely needed to build up confidence in transport modules of sandy sediment models. When models are able to adequately simulate the advectivedispersive transport of conservative tracers, they can be confidently extended to implement more complex biogeochemistry. Here, we provide an illustration of how this could be achieved for our case studies. Model [1] and [3] describe the flow and tracer dynamics in simplified laboratory set-ups. To test the performance of these models, closed-system incubations were conducted with the conservative tracer uranine (Na-fluorescein). A sediment core is incubated with a fixed volume of overlying water, the overlying water is spiked with uranine, and the evolution of the tracer concentration in the overlying water is followed through time. Advective bioirrigation was examined in cylindrical cores to which a single lugworm was added. Similarly, physically-induced exchange was examined in cylindrical chambers with a recirculating flow imposed in the overlying water. The experimental approach is entirely analogous in the lugworm incubations (referred to as "Exp 1") and the benthic chamber experiments (referred to as “Exp 2").

4.2 Model extension: simulation of tracer dynamics in overlying water

Closed-incubation systems have a limited volume of overlying water, and hence, the tracer concentration in the overlying water will change as a result of the exchange with the pore water. Accordingly, in addition to the pore water flow and reactive transport models for the sediment, one needs a suitable model that describes the temporal evolution of the tracer concentration $C^{o w}(t)$ in the overlying water. For an inert tracer, the total tracer inventory in pore water and overlying water should remain constant in time

$$
\begin{aligned}
& V^{o w} C^{o w}(t)+\int_{V^{s}} \phi C^{p w}(r, z, t) d V^{s}= \\
& V^{o w} C_{0}^{o w}+\int_{V^{s}} \phi C_{0}^{p w} d V^{s}
\end{aligned}
$$

where $V^{s}$ is the volume of the sediment and $V^{o w}$ the volume of the overlying water. The initial tracer concentrations in the overlying water and pore water are denoted $C_{0}^{o w}$ and $C_{0}^{p w}$ respectively. The mass balance [13] can be re-arranged into an explicit expression for the tracer concentration in the overlying water column:

$$
C^{o w}(t)=C_{0}^{o w}+\frac{\phi}{V^{o w}} \int_{V^{s}}\left(C_{0}^{p w}-C^{p w}(r, z, t)\right) d V^{s}
$$

The pore water concentration $C^{p w}(r, z, t)$ is provided by the reactive transport model. The integral on the right hand side is numerically evaluated by standard integration procedures. Equation (14) then predicts the evolution of the tracer concentration in the overlying water column. The water column model [14] is coupled to the reactive transport model via the integration coupling variable option in COMSOL Multiphysics 3.2a.

\subsection{Experimental methods}

In both Exp 1 and 2, uranine was used as the tracer, which is a fluorescent, non-toxic, organic compound that belongs to the category of xanthene dyes (Davis et al., 1980; Flury and Wai, 2003). To reduce background fluorescence from organic substances like humic and fulvic acids, and to avoid 
Table 2. Parameter values used in the simulations of the stirred benthic chamber.

\begin{tabular}{lccccc}
\hline Core set-up & & & Run1 & Run2 & $\mathrm{Br}^{-} / \mathrm{O}_{2}$ \\
\hline Core radius & $R_{S}$ & $\mathrm{~cm}$ & 9.5 & 9.5 & 9.5 \\
Sediment height & $H_{S}$ & $\mathrm{~cm}$ & 18.5 & 18.5 & 20 \\
Height overlying water column & $H_{w}$ & $\mathrm{~cm}$ & 10 & 10 & $\mathrm{NA}$ \\
\hline Sediment parameters & & & & & \\
\hline Temperature & $T$ & ${ }^{o} \mathrm{C}$ & 20 & 20 & 15 \\
Salinity & $S$ & - & 0 & 0 & 30 \\
Porosity & $\phi$ & - & 0.37 & 0.37 & 0.5 \\
Permeability & $k$ & $\mathrm{~m}^{2}$ & $1.1 \times 10^{-10}$ & $1.1 \times 10^{-10}$ & $1.0 \times 10^{-11}$ \\
Grain size range & $\bar{r}$ & $\mu \mathrm{m}$ & $300-700$ & $300-700$ & 220 \\
\hline Stirring parameters & & & & & \\
\hline Rotation speed & $\omega$ & $\mathrm{rpm}$ & 40 & 80 & 40 \\
Pressure difference & $p_{0}$ & $\mathrm{~Pa}$ & 3.5 & 14.4 & 3.5 \\
\hline
\end{tabular}

adsorption onto the organic matter coating of sediment particles, clean construction sand was used. Before use, the construction sand was washed thoroughly with demineralised water to further remove any remaining organic matter, and thereby reducing background fluorescence as much as possible. Porosity, permeability, solid phase density, and grain size characteristics are presented in Tables 1 and 2 .

In the incubations, a core with an inner radius $R_{S}$ was first filled with filtered seawater (Exp 1) or tap water (Exp 2). Subsequently, the core was filled to a height $H_{s}$ by adding dry sediment. This was done carefully to prevent the trapping of air bubbles within in the sediment. The height $H_{w}$ of the overlying water was measured. At the beginning of incubation, the overlying water was spiked with uranine stock solution. Subsequently, the uranine fluorescence in the overlying water was measured continuously with a flow through cell. In Exp 1, uranine was measured at $520 \mathrm{~nm}$ using a Turner Quantech Digital Fluorometer (FM 109530-33) with 490 nm as the excitation wavelength. In Exp 2, uranine was determined on a spectrophotometer by measuring absorbance at $490 \mathrm{~nm}$. All experiments were conducted in a darkened room (to prevent degradation of uranine by UV) at a constant temperature of $20^{\circ} \mathrm{C}$. Uranine concentrations are expressed relative to the initial concentration immediately after spiking.

In the lugworm incubations (Exp 1), one specimen of Arenicola marina was introduced in the middle of the sediment surface of the core and was allowed to burrow. Subsequently, the core was left for $48 \mathrm{~h}$ in order to allow the lugworm to acclimatize to the new sediment conditions. It was assumed that after this acclimation period, the lugworm had adopted a representative pumping regime. The position of the lugworm in the core was recorded as the burrowing depth $H_{b}$ (measured from the sediment-water interface). The radius of the injection pocket $R_{b}$ was estimated after the experiment by sectioning the core after the experiment. During the incubation, the overlying water was aerated and continuously kept in motion with a magnetic stirrer.

The set-up of the stirred benthic chambers in Exp 2 is described by Janssen et al. (2005a) and Cook et al. (2006), except for the chamber radius $R_{s}$, which is $95 \mathrm{~mm}$ instead of $100 \mathrm{~mm}$ (Table 2). The distance between the lower surface of the stirrer disc and the sediment was $100 \mathrm{~mm}$. Replicate experiments were carried out with stirrer speeds set to 40 and $80 \mathrm{rpm}$.

\subsection{Results: lugworm bio-irrigation}

The results from two lugworm incubation experiments (Run1 and Run2) are shown in Fig. 7, alongside the output of the corresponding model simulations based upon the water column model [14]. The incubations experiments lasted $30 \mathrm{~h}$ and $17 \mathrm{~h}$ respectively. Note that the model simulations only contain one free adjustable parameter, namely the pumping rate Q. All other parameters are constrained a priori by measurements (Table 1). The correspondence between data and model differed strongly between the two experiments. In the first incubation (Fig. 7a), the simulated tracer evolution showed an excellent correspondence with the experimental data. Fitting the free parameter $\mathrm{Q}$ revealed that throughout the incubation, the lugworm adopted a relatively low, but constant pumping rate of $0.35 \mathrm{ml} \mathrm{min}^{-1}$. In the second incubation however (Fig. 7b), there was a clear mismatch between model simulation and data due to the irregular pumping activity. From fitting the initial slope by adapting Q, the lugworm's pumping rate was estimated to be $1 \mathrm{ml} \mathrm{min}-1$, but after half an hour it stopped pumping, only to resume irrigation after $1.5 \mathrm{~h}$ of inactivity. Subsequently, the tracer concentration decreased with a similar slope as initially, indicating that the pumping rate was similar to the initial rate. These incubations clearly revealed the variability 
Lugworm irrigation [continuous ]

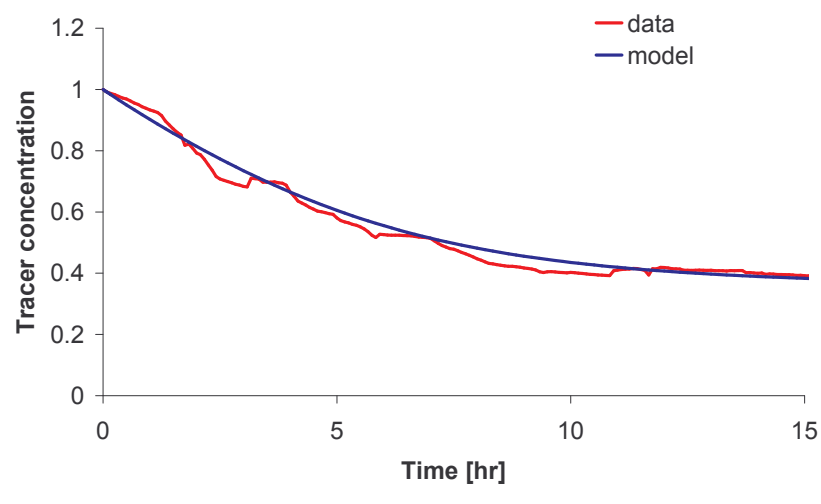

Lugworm irrigation [discontinuous ]

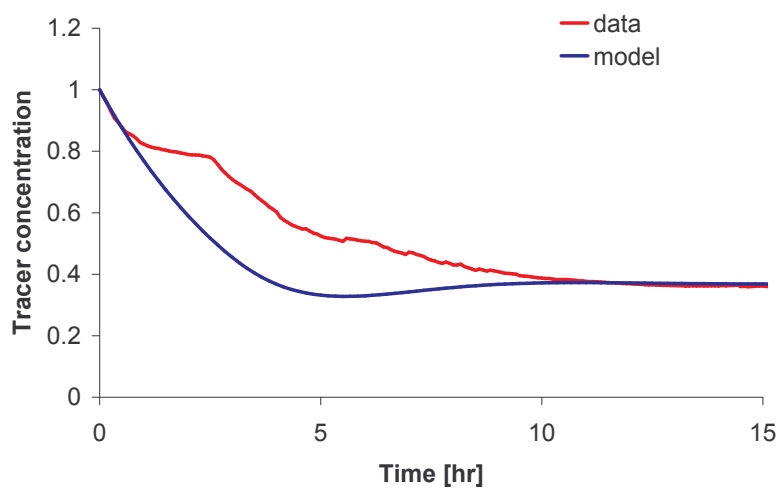

Fig. 7. Validation of the model [1] of the lugworm bio-irrigation. Comparison of the tracer evolution in the overlying water column with model prediction. The tracer concentration is expressed relative to the initial concentration in the overlying water column.

that is inherent to biological activity. In biogeochemical incubations (e.g. oxygen consumption, denitrification), such variability can clearly obstruct the interpretation of measured fluxes and rates. Accordingly, future experimental studies on lugworm bio-irrigation should investigate methods that could reduce temporal variability in lugworm pumping (e.g. finetuning the acclimatization period of freshly collected animals, or using mechanical mimics of lugworm pumping instead of live organisms)

\subsection{Results: benthic chamber exchange}

Again two incubation experiments (at 40 and $80 \mathrm{rpm}$ ) are shown in Fig. 8, alongside the corresponding model output. Note that the model simulations here contain no adjustable parameters. All parameter values are a priori by measurements (Table 2). In both runs, the tracer concentration in the overlying water shows a rapid decrease initially, as tracer rich water is flushed into the sediment and tracer free water flushed out (Fig. 8). After about $5 \mathrm{~h}$, the rate of tracer dilution decreased significantly reflecting the recirculation

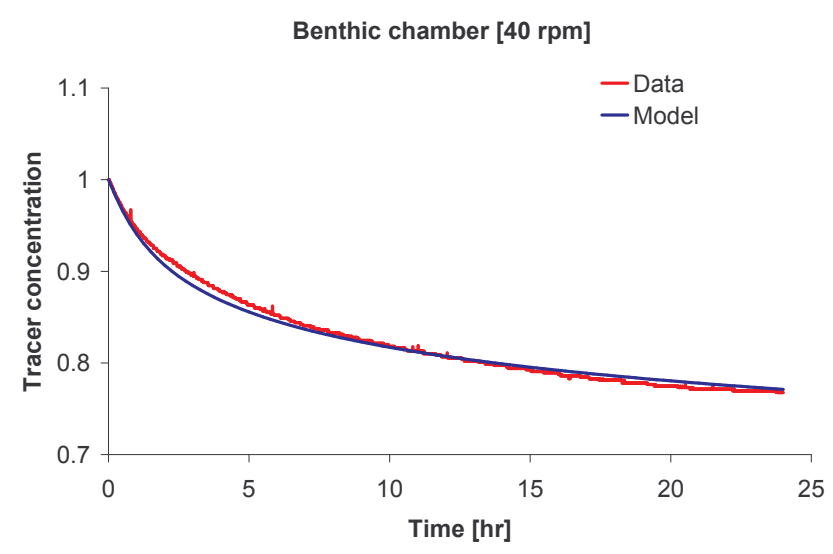

Benthic chamber $[80 \mathrm{rpm}]$

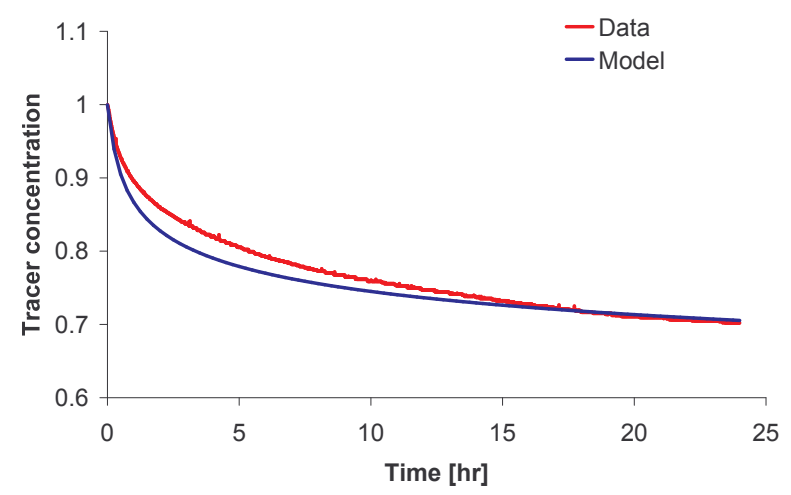

Fig. 8. Validation of the model [3] of the benthic chamber. Comparison of the tracer evolution in the overlying water column with model prediction. The tracer concentration is expressed relative to the initial concentration in the overlying water column.

of tracer through the sediment. The modeled concentration changes of tracer generally shows a good agreement to that measured, with the initial slopes of the measured and modeled tracer concentrations versus time being indistinguishable. The greatest deviation between the measured and modeled results occurred between 1 and $7 \mathrm{~h}$ at the stirring speed of $80 \mathrm{rpm}$, when the model predicted a greater decrease in the tracer concentration compared to that modeled. This deviation needs further investigation in future studies. Possible causes for this discrepancy could be (1) the pressure distribution at the sediment-water interface and (2) the depth dependency of porosity and permeability within the core.

\section{Model application: tracer dynamics of $\mathrm{Br}^{-}$and $\mathrm{O}_{2}$}

The previous section presented "validation simulations": the response of models [1] and [3] was compared to available data sets. In this section, we present a set of "benchmark simulations" that allow a cross-comparison of models, and which could prove useful in future investigations of sandy sediment biogeochemistry. The same two "benchmark" 
situations are simulated using all four models: (a) the timedependent spreading of an inert tracer in the pore water, and (b) the computation of the steady-state distribution of oxygen in the sediment. These benchmark situations are particularly interesting for future work on model validation. The timedependent spreading of a conservative tracer has been experimentally determined in lab studies (e.g. Elliott and Brooks, 1997b) as well as in situ (Precht and Huettel, 2004; Reimers et al., 2004). To date, different inert tracers have been used in studies of advective exchange, such as uranine (Webb and Theodor, 1968; Precht and Huettel, 2004), bromide (Glud et al., 1996), iodide (Reimers et al., 2004) and $\mathrm{NaCl}$ (Salehin et al., 2004). We implemented the diffusion coefficient for bromide in the example simulations. However, the response should be representative for any other conservative tracer. Similarly, the influence of advective transport on oxygen dynamics is a key research topic in biogeochemical studies of sandy sediments. In recent years, planar optodes have been developed that visualize the two-dimensional $\mathrm{O}_{2}$ distribution within sediments. Recently, it has been shown that such optodes have great potential in the study of advective sediments (Precht et al., 2004; Franke et al., 2006). The output of the $\mathrm{O}_{2}$ simulation thus enables a direct comparison with such planar optodes images.

Figures 2 to 6 show the output of the benchmark simulations for all four models. Each time, the flow line pattern, the output of the transient simulation of $\mathrm{Br}^{-}$, and the steady-state simulation of $\mathrm{O}_{2}$ are illustrated. In these simulations, we kept the tracer concentration in the overlying water constant for simplicity. So in contrast to the validation simulations of the previous section, we assume that the water column overlying the sediment is well-mixed and sufficiently large not to be affected by the exchange with the sediment. In laboratory incubation experiments of the previous section, this assumption was clearly invalid due to the limited volume of overlying water. For the inert tracer, the reaction term $R$ vanishes in the reactive transport Eq. (6), and the initial concentration in the pore water is set to zero. In the simulation of oxygen, the consumption rate is modelled by the first-order kinetics

$\mathrm{R}_{\mathrm{O}_{2}}=-k \mathrm{C}_{\mathrm{O}_{2}}$

where the kinetic rate constant is fixed at $k=0.26 \mathrm{~h}^{-1}$. This implies that $\mathrm{O}_{2}$ will be removed from the pore water in approximately $8 \mathrm{~h}$ due to aerobic respiration and re-oxidation of reduced compounds formed in anaerobic pathways of organic matter decomposition. Obviously, the kinetic constant can be readily adapted, and other expressions for the consumption rate may be easily implemented in the model scripts.

\subsection{Lugworm bio-irrigation in a laboratory set-up}

Parameter values were taken for a representative lugworm incubation experiment (incubation " $\mathrm{e}$ " from Rasmussen et al., 1998, which was examined in detail in the modeling papers of Timmermann et al., 2002 and Meysman et al., 2006a parameter values are listed in Table 1). Figure $2 \mathrm{~b}$ shows the typical shape of the predicted flow line pattern: flow lines first "radiate" from injection pocket in all directions, but then curve upwards. Far above the injection pocket, the flow lines align vertically and eventually they all end at the sediment surface. Figure $2 \mathrm{c}$ shows the predicted bromide distribution after two hours of pumping at a constant rate of $1 \mathrm{ml} \mathrm{min}^{-1}$. The injection of tracer at depth creates a subsurface plume of bromide that expands in time. At time zero, this plume starts as a small sphere around injection pocket, but gradually attains an "egg shape" due to the combination of radial and upward flow. When time progresses, the "egg" becomes more and more elongated in the vertical direction. Note that the bromide concentration changes very sharply at the edge of the plume: inside the concentration is nearly that of the overlying water, while outside, the concentration rapidly decreases to zero. This indicates that advection clearly dominates dispersion as a transport mode (higher dispersion would imply a broader fringe). This governance of advection over dispersion in lugworm bio-irrigation was also a central conclusion in the combined experimental-modeling study of Meysman et al. (2006a). After laterally averaging (mimicking the conventional experimental procedure of core sectioning and pore water extraction), the bromide plume will show up as a subsurface maximum in a one-dimensional concentration profile. Such subsurface maxima have been observed in laboratory lugworm incubations (Rasmussen et al., 1998; Timmermann et al., 2002) as well as in field studies of intertidal areas with high lugworm densities (Huettel, 1990).

Figure $2 \mathrm{~d}$ shows the predicted steady-state distribution of oxygen for the same pumping rate. The injection of oxygenated burrow water into the anoxic sediment creates an oxygenated zone around the injection pocket. The oxygenated region has again an egg-shape, though the decline of oxygen concentration is far more gradual than in the transient simulation of bromide. The smaller gradient of the $\mathrm{O}_{2}$ concentration is now due to the interplay of reaction and advective transport: traveling along with a confined water package, the oxygen is gradually consumed within that package. A valid question is how long it takes to establish this steady-state pattern when performing a transient simulation. For the imposed decay constant $k=0.26 \mathrm{~h}^{-1}$, and starting with no oxygen in the pore water, the steady-state pattern of Fig. $2 d$ is reached after approximately six hours of steady pumping (this is about the inverse of the decay constant, i.e., $6 \approx 1 / 0.26$ ).

\subsection{Interaction between lugworm bio-irrigation and up- welling groundwater}

The interaction of lugworm irrigation with the upwelling groundwater flow is similar to the injection/extraction of water from an aquifer with mean regional groundwater flow. 
Javandel and Tsang (1986) provided analytical expressions describing the bell-shaped region of injected water. Our numerical flow simulations (Fig. 3a) also predict that the sediment domain is effectively separated into two separate regions that differ in the origin of their water. Deep layers of sediment are flushed by the upwelling ground water. In the absence of bio-irrigation, this ground water would percolate uniformly towards the sediment-water interface. However, when the lugworm is pumping, it creates a "marine niche" that is filled with overlying sea water (shape of bell-jar turned upside down). The separation between the marine and freshwater zone is shown in Fig. 3 as the bold dashed line. The marine niche serves as an obstacle to the upward flow of the groundwater. The flow lines of the groundwater are diverted around this obstacle. The resulting compression of the flow lines indicates higher pore water velocities. When comparing the flow line pattern in the laboratory core (Fig. 2a) with that in the marine niche (Fig. 3a), one sees that (1) the irrigation zone is compressed sideways, and (2) that burrow water penetrates less deep below the injection pocket. These two effects are due to the "collision" of the upwelling groundwater with the marine niche.

The separation of two water types is nicely illustrated in the simulation of the conservative tracer (Fig. 3b). In the simulation, bromide is added to the overlying water, and thus functions as a tracer for marine water. The zone of bromide saturation has also the shape of an inverted bell-jar, and coincides nicely with the "marine niche" in the flow line pattern in Fig. 3a. In the lower part, near the injection pocket, the bromide concentration gradient is very steep. As the injected water percolates upwards to the sediment surface, side by side with upwelling ground water, hydromechanical dispersion increasingly mixes the two water masses, diminishing the gradient in the bromide concentration. Thus, along the boundary between the two water masses, some moderate mixing of injected sea water and aquifer water takes place. This smoothening of the concentration gradient visible in Fig. $3 \mathrm{c}$ is not a computational artifact due to numerical dispersion: simulations performed with mesh refinement near the separatrix yield essentially the same pattern. Figure 3c shows the steady-state concentration of oxygen. This pattern is essentially the same as in Fig. $2 \mathrm{~d}$ (keeping in mind that the sediment domain is deeper and wider in Fig. 3c).

Our model simulations provide a possible explanation for the observed co-occurrence of denitrification and sulfate reduction within the surface sediment of Ho Bay. The injection of overlying seawater by lugworms creates marine microzones within an otherwise freshwater sediment. Within the marine microniche, nitrate concentrations are low and sulfate concentrations are high, thus enabling sulfate reduction. Outside the microniche, nitrate concentrations are high in the upwelling ground water, thus enabling denitrification upon contact with marine organic matter. When randomly inserting a core into the sediment, one may capture both parts of marine and freshwater environments, and hence, one may ob- serve "apparently" parallel pathways of organic processing, which are in fact spatially separated.

\subsection{Tracer dynamics in a benthic chamber}

The predicted flow line pattern (Fig. 4b) shows both the magnitude (vector arrows) as well as the direction (flow lines) of the pore water flow. Overlying water enters the sediment at the outer part of the core, and in response, pore water is expelled from the central part, in accordance with the dye experiments of Huettel and Gust (1992a). The flow lines show that - theoretically - the whole sediment is affected by flow. However, the magnitude of the velocity vectors rapidly decrease with depth, and so, only the upper $5 \mathrm{~cm}$ of the sediment are truly affected by advection for a given chamber geometry. The transient simulation of bromide (Fig. 4c) was carried out under similar conditions as in model [1]: constant concentration in the overlying water, initially no bromide in the pore water, stirring frequency $40 \mathrm{rpm}$, and a simulated time of $12 \mathrm{~h}$. Close to the outer wall of the chamber, the bromide shows the deepest penetration $(\sim 4 \mathrm{~cm})$. The steadystate distribution of oxygen (Fig. 4d) reveals a penetration depth of $\sim 2 \mathrm{~cm}$ near the outer wall (the concentration decreases to $50 \%$ at the depth of $1 \mathrm{~cm}$ ). This penetration depth matches the range as observed under lab conditions (Huettel and Gust, 1992a), and is much larger than would be expected from diffusive transport alone.

\subsection{Tracer dynamics in a ripple bed}

Rutherford et al. (1995) provide analytical solutions for the penetration and distribution of $\mathrm{O}_{2}$ in triangular ripple beds imposing a known (measured) pressure distribution as a boundary condition at the ripple interface. We provide similar numerical simulations of tracer transport, where the pressure distribution is now derived from a free flow model above the ripple bed. Figures 5a and 6a show the velocity distribution in the water column computed using the laminar and turbulent models, respectively. The laminar flow field establishes immediately, while the turbulent flow pattern is stabilized after passing over a few ripples. As expected, the velocity gradient in the vertical direction is more gradual in the laminar regime as compared to the turbulent case. In the latter case, the velocity profile remains rather plug-like, with a thin transition layer of low velocities above the ripple crest and in between the ripples. The associated flow line patterns within the pore water are illustrated in Figs. 5b, c and Figs. $6 \mathrm{~b}, \mathrm{c}$. The flow line patterns (indicating the direction of the flow velocity) are similar in laminar and turbulent case. The water enters the sediment domain through the upper half of the front slope of the ripple, and leaves the sediment through the lower part of the front slope, through the crest, and through the back slope. The highest outflow velocity is predicted in the narrow zone around the ripple crest. Despite the similarity in flow line patterns, the magnitudes 
of the flow velocities are very different. The total water flux through the surface of a single ripple was 3.6 times lower in the laminar case than in the turbulent one.

Figures $5 \mathrm{~b}$ and $6 \mathrm{~b}$ show the concentration distribution of passive tracer $\left(\mathrm{Br}^{-}\right)$after $12 \mathrm{~h}$ of flushing. Deepest penetration of tracer occurs at the inflow zone in the upper part of the front slope, as advection increases the penetration that would normally be seen under diffusive conditions alone. In the outflow zone near the ripple crest, strong advection is acting against diffusion, reducing the tracer penetration depth to less than $1 \mathrm{~mm}$. However, this value should be treated with caution, as it is actually an artifact of the fixed concentration condition imposed at the SWI. This creates strong gradients, so that that downward diffusion always will counter upward advection. In reality, the outwelling tracer-free water will diminish concentrations in the overlying water, and this will prevent any tracer penetration in the outwelling zone. Despite this, the predicted tracer distribution of bromide shows a good qualitative agreement with tracer patterns observed in flume studies of ripple-induced advection (Huettel et al., 1996; Elliott and Brooks, 1997b).

An important observation is that the tracer penetration depth is markedly different in both regimes (to a depth of $1 \mathrm{~cm}$ in the laminar case and to more than $2 \mathrm{~cm}$ depth in the turbulent case). The steady-state distribution of the reactive tracer (oxygen) is shown in Figs. 5c and 6c. The $\mathrm{O}_{2}$ penetration shows the same trend as for the time-dependent distribution of the conservative tracer. At the upper part of the front slope, the maximum penetration of $\mathrm{O}_{2}$ occurs. The oxic sediment layer is on the order of $0.5 \mathrm{~cm}$ in the laminar case, and $1 \mathrm{~cm}$ in the turbulent case. In the outflow region at the ripple crest, the oxygen penetration depth is reduced, as the water exiting the sediment domain is anoxic. Again, the $\mathrm{O}_{2}$ penetration in the outflow region should not be considered as realistic, because the $\mathrm{O}_{2}$ concentration at the SWI is artificially kept constant in the model. To prevent such an artifact, coupled models of tracer transport in both water column and sediment should be explored.

Clearly, the choice between laminar and turbulent flow strongly influences the predicted tracer pattern within the sediment. Pressure gradients at the sediment-water interface are higher in the turbulent case, leading to higher pore water flow, a 3.6 times larger exchange with the water column, and more conspicuous tracer distribution (deeper tracer penetration in the inflow zone, and reduced penetration in the outflow zones). Turbulent flow over roughness elements like ripples yields a flow circulation and circulation that produces strong boundary gradients. Therefore, the laminar flow model should be expected to significantly underpredict the flux, as discussed in detail in Elliott and Brooks (1997a, b).

However, a pertinent question is whether the results of the turbulent k-epsilon model are realistic and accurate. We do not compare our solutions with data, and hence the correctness of the solutions cannot be ascertained. One indication is the location of the pressure where the main in-flow point is located. Eddy reattachment points, and therefore the pressure maximum, should be located at a distance of four times the bedform height when measured from the ripple crest (Engel, 1981). Our simulation with the k-epsilon model however shows a much closer position to the crest, indicating that the k-epsilon model does not properly capture the circulation in the lee of the bedform, nor the pressure distribution over the ripple interface. Overall the k-epsilon models seems to poorly perform, and other models such as the k-omega formulation (e.g. Yoon and Pattel, 1996) should be explored. Overall, our simulations demonstrate that pore flow within the sediment is very sensitive to the model type chosen for the overlying water, and that close scrutiny of the model output is warranted.

\section{Conclusion and future prospects}

The realisation that advective pore water flow is a significant process in permeable sediments has greatly complicated our conceptualisation of their biogeochemical functioning. There are numerous possible interactions between biological and physical forcings, which are virtually impossible to measure in situ and are hard to simulate experimentally. The modeling approach that is proposed here can be used as a useful and complementary tool alongside dedicated measurements. Models can provide fundamental insight into permeable sediment functioning and biophysical forcings, which is key to identifying important variables to measure and assessing how experiments, representative for in-situ conditions, should be designed. The model comparison presented here shows that model development is promising, but still within an early stage. Clear challenges remain in model development, calibration, validation, and implementation.

\subsection{Challenges in model development}

Our analysis shows that there is a great commonality in the model description of pore water flow and tracer dynamics within permeable sediments. To date, models were typically developed with a specific application in mind, and because of their specific formulation, it is not clear how models relate to each other. Our four case studies illustrate that sandy sediment models have a generic backbone, consisting of the same flow (5) and tracer (6) equations. The principal difference between the model applications concerns the geometry of the sediment-water interface and the pressure conditions that are specified along this boundary. Two approaches are possible: imposing an empirical pressure field at the sediment-water interface, or explicitly modeling the hydrodynamic interaction between overlying water and sediment. A clear challenge for future investigations is to compare these two approaches. For example, empirical pressure relations have been forwarded for stirred benthic chambers (Huettel and Gust, 1992a; Janssen et al., 2005a) and 
triangular ripples (Vittal et al., 1977; Shen et al., 1990). Alternatively, model predictions of this pressure field have also been obtained for benthic chambers (Khalili et al., 1997) and triangular ripples (Cardenas and Wilson, 2007; this work). Future studies should examine how model predicted pressure fields compare to experimental data (as was done in Cardenas and Wilson, 2007 for ripples), and address the cause of possible discrepancies. As indicated in Sect. 6.2, a clear issue is the choice of a proper model for the free flow above the sediment: laminar versus turbulent, and $\mathrm{k}$-epsilon versus k-omega. Previous model applications have assumed by default that the flow is laminar. Yet, under representative field conditions, the flow may be either in the transition from laminar to turbulent, or fully turbulent. Our analysis shows that tracer transport within the pore water is very dependent on the flow regime in the overlying water. The $k-\varepsilon$ turbulence model explored here is only a first step to address this issue, as we found out that the $k-\varepsilon$ does not behave accurately near the sediment water interface. Accordingly, a true challenge will be to find an adequate turbulent flow model that correctly predicts the pressure distribution at the sedimentwater interface of sandy sediments. In this regard, the $k-\omega$ first presented by Yoon and Platel (1996) could be promising. Various efforts are ongoing to implement this model into models for unidirectional flow over ripples (A. Packman, M. B. Cardenas, personal communication).

\subsection{Challenges in model validation}

Model validation is needed to achieve confidence in the behaviour and predictions of the model. Yet, to date, only a limited number of pioneering studies have carried out an in-depth validation of tracer models in permeable sediments (Salehin et al., 2004; Rena and Packman, 2004; Meysman et al., 2006; Cardenas and Wilson, 2007). Ideally, any given model should be confronted with a suite of dedicated tracer experiments, involving different tracers and various experimental conditions. The most simple validation procedure is "water column validation": it involves the monitoring of the evolution of tracer concentrations in the overlying water. Such a procedure was successfully applied in section 5 for the bio-irrigation set-up and benthic chamber. Although a valuable first test for any model, "water column validation" in itself cannot be considered to a sufficient validation. In a sophisticated parameter sensitivity analysis of sediment incubation models, Andersson et al. (2006) recently showed that "water column validation" is a relatively insensitive to most parameters of the sediment model.

Accordingly, methods are needed that allow the measurement of the tracer evolution in the pore water. Until recently, such methods were merely used to get a qualitative picture of the interstitial flow. For example, in the pioneering study of wave action by Webb and Theodor (1968), fluorescein was injected into sand ripples, and the rapid reemergence of this dye into the overlying water was timed. Yet in recent years, sophisticated tracer methods have been developed that allow a more quantitative assessment of the pore water flow velocity. Such methods track the evolution of the tracer through the sediment with an array of multiple sensors (Precht and Huettel, 2004; Reimers et al., 2004). Until now, an in depth comparison between such tracer data and model predictions has only been carried out in a few pioneering studies (e.g. Salehin et al., 2004), leaving models largely untested and datasets under-explored.

\subsection{Challenges in model application}

To date, we only know of a few applications in sandy sediments where a 2-D or 3-D pore water flow model is coupled to a reactive transport model that incorporates complex biogeochemistry, i.e., multiple chemical reactions with nonlinear kinetics (Rena and Packman, 2004, 2005; Cook et al., 2006). Clearly, once the transport part of a given model is thoroughly validated, the reactive part model can be extended. Our model [4] explores the interaction between currents and ripple topography, and in future work this can be extended, to investigate how ripple topography affects sediment processes such as denitrification, nitrification and sulphate reduction. Given that bottom currents and topography change rapidly and frequently, and that they may play an important role in process rates, we suggest that modeling will be vital for the up-scaling of measured local process rates to whole-system budgets in shallow coastal systems.

Acknowledgements. We thank the reviewers M. B. Cardenas and A. Packman, and the editor T. Battin, for their accurate and constructive comments, which greatly improved the manuscript. This research was supported by grants from the EU (NAME project, EVK\#3-CT-2001-00066; COSA project, EVK\#3-CT-2002-00076) and the Netherlands Organization for Scientific Research (NWO PIONIER, 833.02.2002 to J. Middelburg). This is publication 3962 of the Netherlands Institute of Ecology (NIOO-KNAW).

Edited by: T. J. Battin

\section{References}

Aller, R. C.: Transport and Reactions in the Bioirrigated Zone, in: The Benthic Boundary Layer, edited by: Boudreau, B. P. and Jorgensen, B. B., 269-301, Oxford University Press, Oxford, 2001.

Andersson, J. H., Middelburg, J. J., and Soetaert, K.: Identifiability and Uncertainty Analysis of Bio-Irrigation Rates, J. Mar. Res., 64, 407-429, 2006.

Basu, A. J. and Khalili, A.: Computation of Flow Through a FluidSediment Interface in a Benthic Chamber, Phys. Fluids, 11, 1395-1405, 1999.

Bear, J. and Bachmat, Y.: Introduction to Modeling of Transport Phenomena in Porous Media, Kluwer Academic Publishers, Dordrecht, 1991. 
Billerbeck, M., Werner, U., Bosselmann, K., Walpersdorf, E., and Huettel, M.: Nutrient Release From an Exposed Intertidal Sand Flat, Mar. Ecol.-Prog. Ser., 316, 35-51, 2006.

Boudreau, B. P.: The Diffusive Tortuosity of Fine-Grained Unlithified Sediments, Geochim. Cosmochim. Acta, 60, 3139-3142, 1996.

Boudreau, B. P.: Diagenetic Models and Their Implementation, Springer, Berlin, 1997.

Cardenas, M. B., Wilson, J. L.: The Influence of Ambient Groundwater Discharge on Exchange Zones Induced by CurrentBedform Interactions, J. Hydrol., 331, 103-109, 2006.

Cardenas, M. B. and Wilson, J. L.: Hydrodynamics of Coupled Flow Above and Below a Sediment-Water Interface With Triangular Bedforms, Adv. Water Resour., 30, 301-313, 2007.

Cook, P. L. M., Wenzhöfer, F., Rysgaard, S., Galaktionov, O. S., Meysman, F. J. R., Eyre, B. D., Cornwell, J. C., Huettel, M., and Glud, R. N.: Quantification of denitrification in permeable sediments: Insights from a two dimensional simulation analysis and experimental data, Limnol. Oceanogr., Methods, 4, 294-307, 2006.

Davis, S. N., Thompson, G. M., Bentley, H. W., and Stiles, G.: Groundwater Tracers - Short Review, Ground Water, 18, 14-23, 1980.

De Beer, D., Wenzhofer, F., Ferdelman, T. G., Boehme, S. E., Huettel, M., Van Beusekom, J. E. E., Bottcher, M. E., Musat, N., and Dubilier, N.: Transport and Mineralization Rates in North Sea Sandy Intertidal Sediments, Sylt-Romo Basin, Wadden Sea, Limnol. Oceanogr., 50, 113-127, 2005.

Elliott, A. H. and Brooks, N. H.: Transfer of Nonsorbing Solutes to a Streambed With Bed Forms: Theory, Water Resour. Res., 33, 123-136, 1997a.

Elliott, A. H. and Brooks, N. H.: Transfer of Nonsorbing Solutes to a Streambed With Bed Forms: Laboratory Experiments, Water Resour. Res., 33, 137-151, 1997b.

Engel, P.: Length of Flow Separation Over Dunes, Journal of the Hydraulics Division-ASCE, 107, 1133-1143, 1981.

Flury, M. and Wai, N. N.: Dyes as Tracers for Vadose Zone Hydrology, Rev. Geophys., 41, 1002, 2003.

Forster, S., Huettel, M., and Ziebis, W.: Impact of Boundary Layer Flow Velocity on Oxygen Utilisation in Coastal Sediments, Mar. Ecol.-Prog. Ser., 143, 173-185, 1996.

Foster-Smith, R. L.: An Analysis of Water Flow in Tube-living Animals, J. Exp. Mar. Biol. Ecol., 34, 73-95, 1978.

Franke, U., Polerecky, L., Precht, E., and Huettel, M.: Wave Tank Study of Particulate Organic Matter Degradation in Permeable Sediments, Limnol. Oceanogr., 51, 1084-1096, 2006.

Freeze, R. A. and Cherry, J. A: Groundwater, Prentice Hall, Englewood Cliffs, 1979.

Glud, R. N., Forster, S., and Huettel, M.: Influence of Radial Pressure Gradients on Solute Exchange in Stirred Benthic Chambers, Mar. Ecol.-Prog. Ser., 141, 303-311, 1996.

Goharzadeh, A., Khalili, A., and Jorgensen, B. B.: Transition Layer Thickness at a Fluid-Porous Interface, Phys. Fluids, 17, 057102, 2005.

Gust, G. and Harisson, J. T.: Biological Pumps at the SedimentWater Interface: Mechanistic Evaluation of the Alpheid Shrimp Alpheus mackayi and its Irrigation Pattern, Mar. Biol., 64, 71-78, 1981.

Huettel, M.: Influence of the Lugworm Arenicola Marina on Pore- water Nutrient Profiles of Sand Flat Sediments, Mar. Ecol.-Prog. Ser., 62, 241-248, 1990.

Huettel M.: COSA: Coastal Sands as Biocatalytic Filters, Final report, 2006.

Huettel, M. and Gust, G.: Solute Release Mechanisms from Confined Sediment Cores in Stirred Benthic Chambers and Flume Flows, Mar. Ecol.-Prog. Ser., 82, 187-197, 1992a.

Huettel, M. and Gust, G.: Impact of Bioroughness on Interfacial Solute Exchange in Permeable Sediments, Mar. Ecol.-Prog. Ser., 89, 253-267, 1992b.

Huettel, M., Roy, H., Precht, E., and Ehrenhauss, S.: Hydrodynamical Impact on Biogeochemical Processes in Aquatic Sediments, Hydrobiologia, 494, 231-236, 2003.

Huettel, M. and Webster, I. T.: Porewater Flow in Permeable Sediments, in: The Benthic Boundary Layer, Boudreau, B. P. and Jorgensen, B. B., 144-179, Oxford University Press, Oxford, 2001.

Huettel, M., Ziebis, W., and Forster, S.: Flow-Induced Uptake of Particulate Matter in Permeable Sediments, Limnol. Oceanogr., 41, 309-322, 1996.

Huettel, M., Ziebis, W., Forster, S., and Luther, G. W.: Advective Transport Affecting Metal and Nutrient Distributions and Interfacial Fluxes in Permeable Sediments, Geochim. Cosmochim. Acta, 62, 613-631, 1998.

Janssen, F., Faerber, P., Huettel, M., Meyer, V., and Witte, U.: Pore-Water Advection and Solute Fluxes in Permeable Marine Sediments (I): Calibration and Performance of the Novel Benthic Chamber System Sandy, Limnol. Oceanogr., 50, 768-778, 2005a.

Janssen, F., Huettel, M., and Witte, U.: Pore-Water Advection and Solute Fluxes in Permeable Marine Sediments (Ii): Benthic Respiration at Three Sandy Sites With Different Permeabilities (German Bight, North Sea), Limnol. Oceanogr., 50, 779-792, 2005 b.

Javandel, I., Tsang, C. F.: Capture-Zone Type Curves - a Tool for Aquifer Cleanup, Ground Water, 24, 616-625, 1986.

Khalili, A., Basu, A. J., and Huettel, M.: A Non-Darcy Model for Recirculating Flow Through a Fluid- Sediment Interface in a Cylindrical Container, Acta Mech., 123, 75-87, 1997.

Khalili, A., Basu, A. J., Pietrzyk, U., and Jorgensen, B. B.: Advective Transport Through Permeable Sediments: a New Numerical and Experimental Approach, Acta Mech., 132, 221-227, 1999.

Kristensen E.: Impact of Polychaetes (Nereis spp. and Arenicola Marina) on Carbon Biogeochemistry in Coastal Marine Sediments, Geochem. Trans., 2, 92-103, 2001.

Li, M. Z. and Amos, C. L.: Sheet Flow and Large Wave Ripples Under Combined Waves and Currents: Field Observations, Model Predictions and Effects on Boundary Layer Dynamics, Cont. Shelf Res., 19, 637-663, 1999.

Lichtner, P. C.: Continuum Formulation of MulticomponentMultiphase Reactive Transport, in: Reactive Transport in Porous Media, edited by: Lichtner, P. C., Steefel, C. C., and Oelkers, E. H., 1-82, The Mineralogical Society of America, Washington, 1996.

Massel, S. R., Przyborska, A., and Przyborski, M.: Attenuation of Wave-Induced Groundwater Pressure in Shallow Water. Part 1, Oceanologia, 46, 383-404, 2004.

Meile, C., Tuncay, K., and Van Cappellen, P.: Explicit Representation of Spatial Heterogeneity in Reactive Transport Models: Application to Bioirrigated Sediments, J. Geochem. Explor., 78-9, 231-234, 2003. 
Meysman, F. J. R., Galaktionov, E. S., and Middelburg, J. J.: Irrigation Patterns in Permeable Sediments Induced by Burrow Ventilation: a Case Study of Arenicola Marina, Mar. Ecol.-Prog. Ser., 303, 195-212, 2005.

Meysman, F. J. R., Galaktionov, O. S., Gribsholt, B., and Middelburg, J. J.: Bio-irrigation in Permeable Sediments: Advective Pore Water Transport induced by Burrow Ventilation, Limnol. Oceanogr., 51, 142-156, 2006a.

Meysman, F. J. R., Galaktionov, O. S., Gribsholt, B., and Middelburg, J. J.: Bio-irrigation in Permeable Sediments: An assessment of model complexity, J. Mar. Res., 64, 589-627, $2006 \mathrm{~b}$.

Oelkers, E. H.: Physical and Chemical Properties of Rocks and Fluids for Chemical Mass Transport Calculations, in: Reactive Transport in Porous Media, edited by: Lichtner, P. C., Steefel, C. C., and Oelkers, E. H., 131-191, The Mineralogical Society of America, Washington, 1996.

Postma, D.: NAME: Nitrate from Aquifers and influences on carbon cycling in Marine Ecosystems. Final report, 2005.

Precht, E., Franke, U., Polerecky, L., and Huettel, M.: Oxygen Dynamics in Permeable Sediments With Wave-Driven Pore Water Exchange, Limnol. Oceanogr., 49, 693-705, 2004.

Precht, E. and Huettel, M.: Advective Pore-Water Exchange Driven by Surface Gravity Waves and Its Ecological Implications, Limnol. Oceanogr., 48, 1674-1684, 2003.

Precht, E. and Huettel, M.: Rapid Wave-Driven Advective Pore Water Exchange in a Permeable Coastal Sediment, J. Sea Res., 51, 93-107, 2004.

Rasmussen, A. D., Banta, G. T., and Andersen, O.: Effects of Bioturbation by the Lugworm Arenicola marina on Cadmium Uptake and Distribution in Sandy Sediments, Mar. Ecol.-Prog. Ser., 164, 179-188, 1998.

Reimers, C. E., Stecher, H. A., Taghon, G. L., Fuller, C. M., Huettel, M., Rusch, A., Ryckelynck, N., and Wild, C.: In Situ Measurements of Advective Solute Transport in Permeable Shelf Sands, Cont. Shelf Res., 24, 183-201, 2004.

Ren, J. H., Packman, A. I.: Modeling of Simultaneous Exchange of Colloids and Sorbing Contaminants Between Streams and Streambeds, Environ. Sci. Technol., 38, 2901-2911, 2004.

Rena, J. H. and Packman, A. I.: Coupled Stream-Subsurface Exchange of Colloidal Hematite and Dissolved Zinc, Copper, and Phosphate, Environ. Sci. Technol., 39, 6387-6394, 2005.

Riedl, R. J., Huang, N., and Machan, R.: The subtidal pump: a mechanism of interstitial water exchange by wave action, Mar. Biol., 13, 210-221, 1972.

Riisgard, H. U. and Banta, G. T.: Irrigation and Deposit Feeding by the Lugworm Arenicola Marina, Characteristics and Secondary Effects on the Environment. A Review of Current Knowledge, Vie Milieu, 48, 243-257, 1998.

Riisgard, H. U., Berntsen, I., and Tarp, B.: The Lugworm (Arenicola marina) Pump: Characteristics, Modelling and Energy Cost, Mar. Ecol.-Prog. Ser., 138, 149-156, 1996.

Rusch, A. and Huettel, M.: Advective Particle Transport Into Permeable Sediments - Evidence From Experiments in an Intertidal Sandflat, Limnol. Oceanogr., 45, 525-533, 2000.
Rutherford, J. C., Boyle, J. D., Elliott, A. H., Hatherell, T. V. J., and Chiu, T. W.: Modeling Benthic Oxygen-Uptake by Pumping, J. Environ. Eng.-ASCE, 121, 84-95, 1995.

Salehin, M., Packman, A. I., and Paradis, M. : Hyporheic Exchange With Heterogeneous Streambeds: Laboratory Experiments and Modeling, Water Resour. Res., 40, W11504, doi:10.1029/2003WR002567, 2004.

Savant, S. A., Reible, D. D., and Thibodeaux, L. J.: ConvectiveTransport Within Stable River Sediments, Water Resour. Res., 23, 1763-1768, 1987.

Shen, H. W., Fehlman, H. M., and Mendoza, C.: Bed Form Resistances in Open Channel Flows, J. Hydraul. Eng.-Asce, 116, 799-815, 1990.

Shum, K. T.: Wave-Induced Advective Transport Below a Rippled Water-Sediment Interface, J. Geophys. Res.-Oceans, 97, 789808, 1992.

Shum, K. T. and Sundby, B.: Organic Matter Processing in Continental Shelf Sediments - the Subtidal Pump Revisited, Mar. Chem., 53, 81-87, 1996.

Thibodeaux, L. J. and Boyle, J. D.: Bedform-Generated Convective-Transport in Bottom Sediment, Nature, 325, 341343, 1987.

Timmermann, K., Banta, G. T., Larsen, J., and Andersen, O.: Modelling Particle and Solute Transport in Sediments Inhabited by Arenicola Marina. Effects of Pyrene on Transport Processes, Vie Milieu, 53, 187-200, 2003.

Timmermann, K., Christensen, J. H., and Banta, G. T.: Modeling of Advective Solute Transport in Sandy Sediments Inhabited by the Lugworm Arenicola Marina, J. Mar. Res., 60, 151-169, 2002.

Vanderloeff, M. M. R.: Wave Effects on Sediment Water Exchange in a Submerged Sand Bed, Neth. J. Sea Res., 15, 100-112, 1981.

Vittal, N., Raju, K. G. R., and Garde, R. J.: Resistance of 2 Dimensional Triangular Roughness, J. Hydraul. Res., 15, 19-36, 1977.

Wheatcroft, R. A.: Temporal Variation in Bed Configuration and One-Dimensional Bottom Roughness at the Mid-Shelf Stress Site, Cont. Shelf Res., 14, 1167-1190, 1994.

Webb, A. P. and Eyre, B. D.: Effect of Natural Populations of Burrowing Thalassinidean Shrimp on Sediment Irrigation, Benthic Metabolism, Nutrient Fluxes and Denitrification, Mar. Ecol.Prog. Ser., 268, 205-220, 2004.

Webb, J. E. and Theodor, J.: Irrigation of Submerged Marine Sands Through Wave Action, Nature, 220, 682-683, 1968.

Webb, J. E. and Theodor, J. L.: Wave-Induced Circulation in Submerged Sands, J. Mar. Biol. Assoc. UK, 52, 903-914, 1972.

Yoon, J. Y. and Patel, V. C.: Numerical Model of Turbulent Flow Over Sand Dune, J. Hydraul. Eng.-Asce, 122, 10-18, 1996.

Zhou, D. H. and Mendoza, C.: Flow Through Porous Bed of Turbulent Stream, J. Eng. Mech.-ASCE, 119, 365-383, 1993.

Ziebis, W., Forster, S., Huettel, M., and Jorgensen, B. B.: Complex Burrows of the Mud Shrimp Callianassa Truncata and Their Geochemical Impact in the Sea Bed, Nature, 382, 619-622, 1996. 